\title{
Mechanical effects of optical resonators on driven trapped atoms: Ground-state cooling in a high-finesse cavity
}

\author{
Stefano Zippilli ${ }^{1}$ and Giovanna Morigi ${ }^{2}$ \\ ${ }^{1}$ Abteilung für Quantenphysik, Universität Ulm, D-89069 Ulm, Germany \\ ${ }^{2}$ Grup d'Optica, Departament de Fisica, Universitat Autònoma de Barcelona, 08193 Bellaterra, Spain
}

(Received 8 August 2005; published 14 November 2005)

\begin{abstract}
We investigate theoretically the mechanical effects of light on atoms trapped by an external potential, whose dipole transition couples to the mode of an optical resonator and is driven by a laser. We derive an analytical expression for the quantum center-of-mass dynamics, which is valid in presence of a tight external potential. This equation has broad validity and allows for a transparent interpretation of the individual scattering processes leading to cooling. We show that the dynamics is a competition of the mechanical effects of the cavity and of the laser photons, which may mutually interfere. We focus on the good-cavity limit and identify novel cooling schemes, which are based on quantum interference effects and lead to efficient ground-state cooling in experimentally accessible parameter regimes.
\end{abstract}

DOI: $10.1103 /$ PhysRevA.72.053408

PACS number(s): $32.80 . P j, 42.50 . V k$

\section{INTRODUCTION}

Atom cooling by photon scattering is achieved by enhancing the rate of scattering processes that dissipate motional energy, thereby exploiting the conservation of internal and mechanical energy in the interaction between atoms and electromagnetic field [1]. The atomic scattering cross section can be significantly modified by the coupling to an optical resonator, which acts both on the internal and on the external degrees of freedom. Hence, the scattering properties can be tailored, allowing one to achieve efficient cooling also for atoms and molecules which may not offer a convenient configuration in free space $[2,3]$. This principle is at the basis of cooling by means of an optical resonator. Indeed, the mechanical effects on atoms coupled to an optical resonator are the object of several experimental [4-12] and theoretical [3,13-22] investigations, which aim at developing a systematic understanding of these complex dynamics both for its fundamental aspects and for the perspective of a high degree of control of complex systems with scalable number of degrees of freedom.

In this work we investigate the cooling dynamics of atoms inside optical resonators, when their center-of-mass motion is tightly confined by an external potential, like, for instance, a dipole $[7,23]$ or an ion trap [11,24-26]. We consider the situation where an atomic optical dipole transition is driven by a laser and by a cavity resonator, as sketched in Fig. 1, and discuss in detail the results presented in [27]. In particular, we show the detailed derivation of the rate equation discussed in [27]. This equation has broad validity, which is supported by numerical checks, and allows for a transparent interpretation of the individual scattering processes leading to cooling. Moreover, in the corresponding parameter regimes it reproduces the results reported in $[13,15]$.

In this paper we mostly focus on the good-cavity limit. In this regime we discuss when efficient cooling into the potential ground state can be achieved. In particular, we show that in experimentally accessible parameter regimes one may obtain almost unit ground-state occupation, even when the natural linewidth of the dipole transition would not allow for ground-state cooling in free space. Efficient ground-state cooling is often found by exploiting interference effects, arising from phase correlation between the laser and field scattered by the atom into the resonator. Most of these interference effects are due to the discrete nature of the spectrum of the center-of-mass motion, which is trapped by a harmonic potential. Hence, the dynamics here studied differs substantially from that of the cooling of free atoms inside cavities $[3,14,17-19]$. Such interference effects are at the basis of novel cooling schemes, some of which have been identified in [27] and which are discussed in detail in the present work.

This article is organized as follows. In Sec. II some preliminary considerations are made. In Sec. III the model is introduced and the basic equations for the motion are obtained. In Sec. IV we discuss the dynamics of cooling from the rate equation we obtain and review previous results presented in the literature. In Sec. V novel cooling schemes are

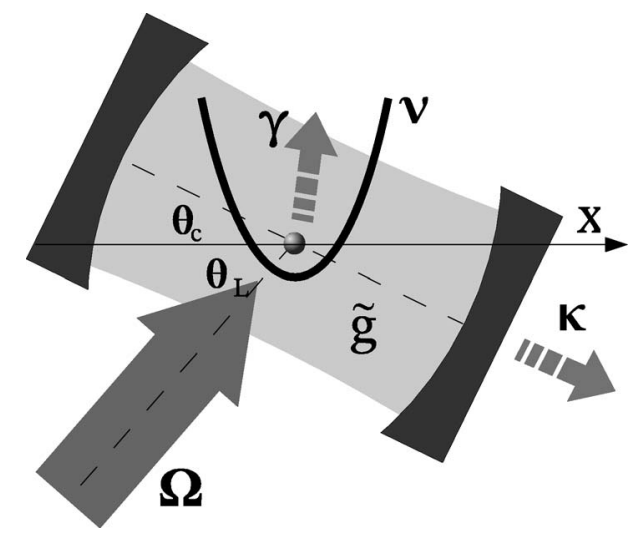

FIG. 1. An atom is confined by an harmonic potential of frequency $\nu$ inside an optical resonator. A mode of the resonator couples with strength $\widetilde{g}$ to the dipole, which is driven transversally by a laser at Rabi frequency $\Omega$. The system dissipates by spontaneous emission of the atomic excited state at rate $\gamma$ and by cavity decay at rate $\kappa$. The other parameters are discussed in Sec. III. 


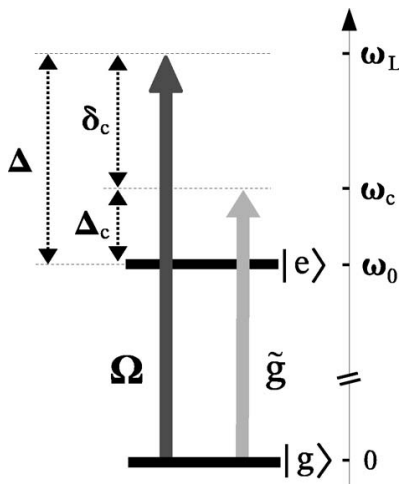

FIG. 2. Sketch of the internal levels $|g\rangle$ and $|e\rangle$ of the atomic dipole transition, driven by a laser and a cavity mode with coupling strengths $\widetilde{g}$ and $\Omega$, respectively. The arrows show the cavity and laser frequency with respect to the dipole frequency. Here, $\Delta$ and $\delta_{c}$ are the detunings of atom and cavity, respectively, from the laser frequency, and $\Delta_{c}$ is the detuning of the atomic transition from the cavity frequency. The frequencies of the atomic transition $\left(\omega_{0}\right)$, of the cavity mode $\left(\omega_{c}\right)$, and of the laser field $\left(\omega_{L}\right)$ are indicated in the vertical scale.

presented, whose dynamics are due to quantum correlations which are established in the good cavity limit. In Sec. VI the results are reported: The cooling efficiencies in the various parameter regimes are discussed and compared. In Sec. VII the conclusions are drawn. The Appendixes report detailed calculations at the basis of the equations derived in Secs. III-V.

\section{MECHANICAL EFFECTS OF CAVITY AND LASER ON THE ATOMIC MOTION}

In this section we make some physical considerations, in order to provide insight into the results presented in the rest of the paper. The scattering cross section of the bare atom is usually very informative about the cooling process [28]. When the atomic transition is driven in saturation, this analysis is more conveniently done in the dressed-state picture $[19,29,30]$. For this purpose, we first assume that the atom is fixed at the position $x$, such that the coupling constant to the cavity mode is $\widetilde{g}=g(x)$. The dipole level scheme and the relevant parameters are shown in Fig. 2. We denote by $\left|g, n_{c}\right\rangle$ and $\left|e, n_{c}\right\rangle$ the states of the system, where $|g\rangle,|e\rangle$ are the ground and excited states of the atomic dipole and $\left|n_{c}\right\rangle$ the number of photons of the cavity mode. In the situation in which the atom is strongly coupled to the cavity mode and weakly pumped by the laser, the states which are relevantly involved into the dynamics are $\left|g, 0_{c}\right\rangle$ and the dressed states

$$
\begin{aligned}
& |+\rangle=\sin \vartheta\left|g, 1_{c}\right\rangle+\cos \vartheta\left|e, 0_{c}\right\rangle, \\
& |-\rangle=\cos \vartheta\left|g, 1_{c}\right\rangle-\sin \vartheta\left|e, 0_{c}\right\rangle,
\end{aligned}
$$

with

$$
\tan \vartheta=\widetilde{g} /\left(-\Delta_{c} / 2+\sqrt{\widetilde{g}^{2}+\Delta_{c}^{2} / 4}\right)
$$

and $\Delta_{c}$ the detuning between cavity mode and atom. Setting the energy of $\left|g, 0_{c}\right\rangle$ at zero, the frequencies of the states $| \pm\rangle$ are
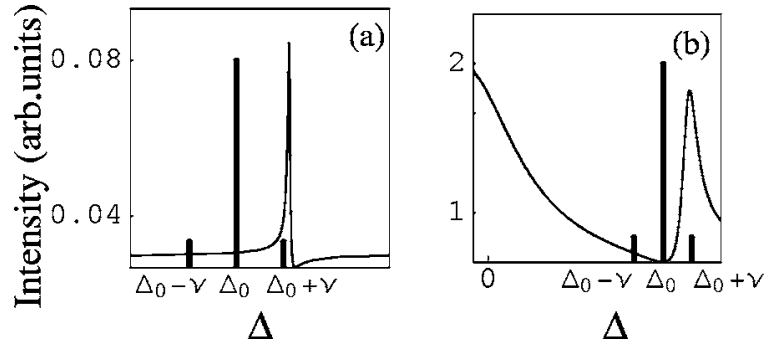

FIG. 3. Excitation spectrum as a function of the laser detuning $\Delta$ in the reference frame of the atom. Here, $\tilde{g}=0.5 \gamma$ and $\kappa=0.01 \gamma$. In (a) $\Delta_{c}=-10 \gamma$ and (b) $\Delta_{c}=1.2 \gamma$. The vertical bars indicate the frequency $\Delta_{0}$ of the carrier (central line) and of the red and blue sideband transitions, $\Delta_{0}+\nu$ and $\Delta_{0}-\nu$, respectively, when the laser is set at $\Delta=\Delta_{0}$ and the trap frequency $\nu=0.2 \gamma$. In (a) $\Delta_{0} \sim \Delta_{c}-\nu$; in (b) $\Delta_{0}=\Delta_{c}$-namely, cavity mode and laser are resonant. See text.

$$
\lambda_{ \pm}=-\Delta_{c} / 2 \pm \sqrt{\tilde{g}^{2}+\Delta_{c}^{2} / 4}
$$

and the respective linewidths are $\gamma_{+} \sim \kappa \sin ^{2} \vartheta+\gamma \cos ^{2} \vartheta$ and $\gamma_{-} \sim \kappa \cos ^{2} \vartheta+\gamma \sin ^{2} \vartheta$, where $\gamma$ is the linewidth of the dipole transition and $\kappa$ the cavity decay rate. The weak laser probe couples the dressed states $\left|g, 0_{c}\right\rangle$ and $\left|e, 0_{c}\right\rangle$.

Signatures of the dressed states are, for instance, the resonances in the rate of photon scattering as obtained by scanning the probe laser through atomic resonance. This situation is depicted in Fig. 3. Here, the curve has been evaluated for a good resonator-namely, $\kappa \ll \gamma, \tilde{g}$ and $\Delta_{c} \neq 0$. For these parameters the linewidth of one of the two resonances is narrower than the natural linewidth of the dipole. Moreover, when the probe laser is resonant with the cavity mode, the spectrum exhibits a minimum, which reaches zero for $\kappa=0$; namely, no photons are scattered. This behavior is due to an interference effect between laser and cavity resonator, such that there is no radiation scattered by the atom, as it is at a point where the two fields, laser and cavity, mutually cancel [31-33].

We now consider the center-of-mass motion of an atom in a harmonic oscillator and first assume that the mechanical effects are only due to the laser, while the cavity wave vector is orthogonal to the motional axis. In this regime, the motion gives rise to a modulation of the laser frequency at the trap frequency $\nu$. In the regime of strong confinement (LambDicke regime) this gives rise to two sidebands of the carrier-i.e., the laser frequency. The carrier and sideband positions are indicated by the vertical bars in Fig. 3 in the reference frame of the atom. The central bar is the carrier. The bar at the right (left) of the carrier corresponds to a transition which lowers (raises) the atomic vibrational excitation by one phonon-namely, the so-called red (blue) sideband transition. These two components are out of phase with respect to the carrier. In the limit in which the atomic motion weakly perturbs the internal and cavity dynamics, the scattering along the sidebands is proportional to the corresponding value of the excitation spectrum. Cooling is thus obtained by realizing a large gradient between scattering rates along the sidebands. Figures 3(a) and 3(b) show two possible scenarios, which are discussed in this paper. Case (a) corresponds to use the narrow resonance for implementing side- 


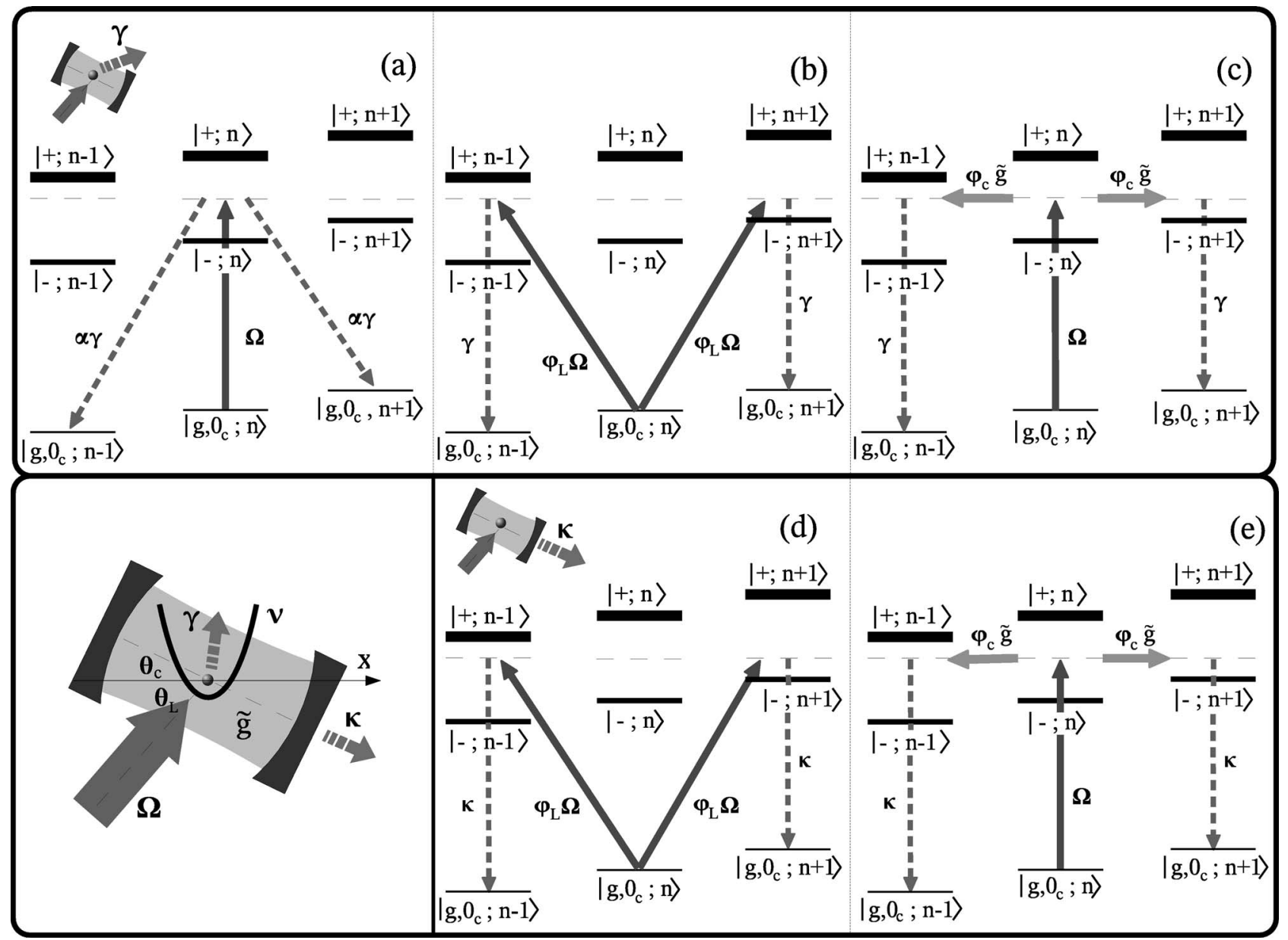

FIG. 4. Scattering processes leading to a change of the vibrational number by one phonon. The states $\left|g, 0_{c} ; n\right\rangle,| \pm ; n\rangle$ are the cavity-atom dressed states at phonon number $n$. Processes (a), (b), and (c) describe scattering of a laser photon by spontaneous emission. They prevail in good resonators, for $\kappa \ll \tilde{g}, \gamma$. Processes (d) and (e) describe scattering of a laser photon by cavity decay. They prevail in bad resonators, for $\gamma \ll \widetilde{g}, \kappa$. The parameters $\alpha, \varphi_{c}$, and $\varphi_{L}$ emerge from the mechanical effects of light and are defined in Sec. III B.

band cooling with the dressed states [15]. This scenario is obtained by choosing a large value of $\left|\Delta_{c}\right|$ and setting the detuning between the cavity and laser equal to the trap frequency, such that the red sideband absorption falls at the center of the narrow resonance. This case has been studied in [15]. In case (b) a large gradient is achieved by exploiting the interference profile arising when laser and cavity are resonant.

The dressed-state picture, as obtained by neglecting the motion of the atom, can be also applied to get some insight into the cooling dynamics when cooling is due only to the resonator forces or to both laser and resonator. Nevertheless, it does not explain other cooling dynamics, which we discuss in this article and which are due to correlations in the gradients of the fields over the atomic wave packet. At this purpose one has to consider also the quantum motion.

Figure 4 summarizes the basic scattering processes determining the cooling dynamics in the basis $\left|g, 0_{c} ; n\right\rangle,| \pm ; n\rangle$, where $n$ is the number of excitations of the center-of-mass harmonic oscillator. The process shown in Fig. 4(a) describes the absorption of a laser photon and spontaneous emission, whereby the change in the center-of-mass state is due to the recoil induced by the spontaneously emitted photon. The scattering rate is scaled by the geometric factor $\alpha$ and is found after averaging over the solid angle of photon emission into free space. This contribution is diffusive, as the motion can be scattered into a higher or lower vibrational state with probabilities depending on the overlap integrals between the initial and final motional states after a photon recoil. In Sec. V we discuss the parameter regime in which this contribution can be suppressed by an interference effect in the dressed states absorption.

The processes depicted in Figs. 4(b) and 4(c) describe scattering of a laser photon by spontaneous emission where the motion is changed by mechanical coupling to the laser $[(b)]$ and to the cavity [(c)] field. Since the final state of the two scattering processes is the same, they interfere. In addition, each term is composed of multiple excitation paths and can vanish in some parameter regimes. In Sec. V we discuss interference effects in these two terms.

The processes depicted in Figs. 4(d) and 4(e) describe the scattering of a laser photon by cavity decay, where the motion is changed by mechanical coupling to the laser [(d)] and to the cavity $[(\mathrm{e})]$ field. The scattered photon is transmitted through the cavity mirrors into the external modes, and therefore these two processes do not interfere with the ones 
above discussed, but add up coherently with one another. In Sec. IV C we discuss parameter regimes where interference effects in these two terms relevantly affect the dynamics. The general dynamics is a competition of all these processes and will be discussed in detail in the following sections.

\section{THE MODEL}

\section{A. Basic equations}

We consider an atom of mass $M$, which is confined by a harmonic potential of frequency $\nu$ inside an optical resonator. The relevant center-of-mass dynamics is along the $x$ axis, while the degrees of freedom of the transverse motion have been traced out, assuming that the transverse confinement is much steeper. Later on we discuss how the treatment can be generalized to three-dimensional motion. The atom internal degrees of freedom, which are relevant to the dynamics, are the ground state $|g\rangle$ and the excited state $|e\rangle$, constituting a dipole transition at frequency $\omega_{0}$ and linewidth $\gamma$. The dipole couples with a cavity mode at frequency $\omega_{c}$ and with a laser at frequency $\omega_{L}$, whose wave vectors $\mathbf{k}_{c}$ and $\mathbf{k}_{L}$ form the angle $\theta_{c}$ and $\theta_{L}$, respectively, with the $x$ axis. The system is sketched in Figs. 1 and 2. We denote by $\rho$ the density matrix for the atom and resonator degrees of freedom in the reference frame rotating at the laser frequency. The density matrix $\rho$ obeys the master equation

$$
\frac{\partial}{\partial t} \rho=\frac{1}{\mathrm{i} \hbar}[H, \rho]+\mathcal{L}_{s} \rho+\mathcal{K} \rho \equiv \mathcal{L} \rho,
$$

where $\mathcal{L}$ is the Liouvillian describing the total dynamics. Here, the Hamiltonian $H$ is

$$
H=H_{\text {mec }}+H_{\text {at }}+H_{\text {cav }}+H_{\text {at-cav }}+H_{L},
$$

where the terms describing the coherent dynamics in absence of coupling with the electromagnetic (e.m.) field are

$$
\begin{gathered}
H_{\mathrm{mec}}=\frac{p^{2}}{2 M}+\frac{1}{2} M \nu^{2} x^{2}, \\
H_{\mathrm{at}}=-\hbar \Delta \sigma^{\dagger} \sigma, \\
H_{\mathrm{cav}}=-\hbar \delta_{c} a^{\dagger} a .
\end{gathered}
$$

Here, $x$ and $p$ are the position and momentum of the center of mass; $\sigma=|g\rangle\langle e|$, and $\sigma^{\dagger}$ its adjoint; $a$ and $a^{\dagger}$ are the annihilation and creation operators of a cavity photon; $\Delta=\omega_{L}-\omega_{0}$ and $\delta_{c}=\omega_{L}-\omega_{c}$ are the detunings of the laser from the dipole and from the cavity frequency, respectively, such that

$$
\Delta_{c}=\Delta-\delta_{c}
$$

The terms

$$
\begin{gathered}
H_{\text {at-cav }}=\hbar g \cos \left(k x \cos \theta_{c}+\phi\right)\left(a^{\dagger} \sigma+a \sigma^{\dagger}\right), \\
H_{L}=\hbar \Omega\left(e^{\mathrm{i} k x \cos \theta_{L}} \sigma^{\dagger}+\text { H.c. }\right)
\end{gathered}
$$

describe the radiative couplings of the dipole with the cavity mode and laser, respectively, where $g$ is the cavity-mode vacuum Rabi frequency and $\Omega$ the Rabi frequency for the coupling with the laser, $\phi$ is a phase, and $k$ is the modulus of the wave vector $\left(\left|\mathbf{k}_{L}\right| \approx\left|\mathbf{k}_{c}\right| \approx \omega_{0} / c=k\right)$.

The superoperators $\mathcal{K}$ and $\mathcal{L}_{s}$ in Eq. (4) describe the cavity decay and dipole spontaneous emission into the modes external to the resonator, respectively, and are

$$
\begin{aligned}
\mathcal{K} \rho & =\frac{\kappa}{2}\left(2 a \rho a^{\dagger}-a^{\dagger} a \rho-\rho a^{\dagger} a\right), \\
\mathcal{L}_{s} \rho & =\frac{\gamma}{2}\left(2 \sigma \tilde{\rho} \sigma^{\dagger}-\sigma^{\dagger} \sigma \rho-\rho \sigma^{\dagger} \sigma\right),
\end{aligned}
$$

where $\kappa$ is the cavity decay rate due to the finite transmission at the mirrors and

$$
\widetilde{\rho}=\int_{-1}^{1} \mathrm{~d} \cos \theta_{0} \mathcal{N}\left(\theta_{0}\right) e^{-\mathrm{i} k \cos \theta_{0} x} \rho e^{\mathrm{i} k \cos \theta_{0} x}
$$

describes the events in which the atomic motion recoils by emission of a photon at the angle $\theta_{0}$ with the trap axis with probability $\mathcal{N}\left(\theta_{0}\right) \mathrm{d} \cos \theta_{0}$. Note that $\mathcal{N}\left(\theta_{0}\right)$ must be evaluated taking into account the geometry of the setup.

For later convenience we introduce the annihilation and creation operators $b$ and $b^{\dagger}$ of a quantum of vibrational energy, such that

$$
\begin{gathered}
x=\sqrt{\hbar / 2 M \nu}\left(b^{\dagger}+b\right), \\
p=\mathrm{i} \sqrt{\hbar M \nu / 2}\left(b^{\dagger}-b\right),
\end{gathered}
$$

and the Hamiltonian term (6) can be rewritten as

$$
H_{\mathrm{mec}}=\hbar \nu\left(b^{\dagger} b+\frac{1}{2}\right) \text {. }
$$

We denote by $|n\rangle$ the eigenstates of $H_{\text {mec }}$ at the eigenvalue $(n+1 / 2) \hbar \nu$ and introduce the Lamb-Dicke parameter

$$
\eta=k \sqrt{\frac{\hbar}{2 M \nu}}
$$

which scales the mechanical coupling of radiation with the atomic motion.

\section{B. Reduced equation for the center-of-mass dynamics in the Lamb-Dicke limit}

We assume the Lamb-Dicke regime-namely, the atom is localized on a length scale which is much smaller than the light wave length - and identify in the Lamb-Dicke parameter $\eta$ the perturbative parameter, which allows us to treat the coupling of the external degrees of freedom with the cavity and the atom internal degrees of freedom in perturbation theory $[34,35]$. We apply the formalism first applied in [35] and then further developed in [36-38]. Below we summarize some steps.

At zero order in the Lamb-Dicke parameter the center of mass is decoupled from the internal and cavity degrees of freedom. In fact, denoting by $\mathcal{L}_{0}=\left.\mathcal{L}\right|_{\eta=0}$ the Liouvillian at zeroth order in the expansion, this can be decomposed into 
the sum of a term acting over the external and over the cavity and dipole degrees of freedom-namely,

$$
\mathcal{L}_{0}=\mathcal{L}_{0 E}+\mathcal{L}_{0 I}
$$

where

$$
\begin{gathered}
\mathcal{L}_{0 E} \rho=\frac{1}{\mathrm{i} \hbar}\left[H_{\mathrm{mec}}, \rho\right], \\
\mathcal{L}_{0 I} \rho=\frac{1}{\mathrm{i} \hbar}\left[H_{\mathrm{at}}+H_{\mathrm{cav}}+H_{0 \mathrm{at}-\mathrm{cav}}+H_{0 L}, \rho\right]+\mathcal{K} \rho+\mathcal{L}_{0 s} \rho
\end{gathered}
$$

and where the Hamiltonian interaction

$$
\begin{gathered}
H_{0 \text { at-cav }}=\hbar \tilde{g}\left(a^{\dagger} \sigma+a \sigma^{\dagger}\right), \\
H_{0 L}=\hbar \Omega\left(\sigma^{\dagger}+\sigma\right)
\end{gathered}
$$

and the Liouvillian for the atomic spontaneous emission,

$$
\mathcal{L}_{0 s} \rho=\frac{\gamma}{2}\left(2 \sigma \rho \sigma^{\dagger}-\sigma^{\dagger} \sigma \rho-\rho \sigma^{\dagger} \sigma\right)
$$

appear at zeroth order in the expansion in $\eta$. The term

$$
\tilde{g}=g \cos \phi
$$

is the zeroth-order atom-cavity coupling strength.

The spectrum of $\mathcal{L}_{0}$ is $\lambda=\lambda_{I}+\lambda_{E}$, where $\lambda_{I}$ are the eigenvalues of $\mathcal{L}_{0 I}$ and $\lambda_{E}$ are the eigenvalues of $\mathcal{L}_{0 E}$. The stationary state is a right eigenstate at eigenvalue zero, as it fulfills the secular equation $\mathcal{L}_{0} \rho=0$ [39]. The corresponding eigenspace is spanned, for instance, by the eigenvectors $\rho_{n}=\rho_{\mathrm{St}}$ $\otimes|n\rangle\langle n|$, where $\rho_{\text {St }}$ fullfills the equation

$$
\mathcal{L}_{0 I} \rho_{\mathrm{St}}=0,
$$

while the operator $|n\rangle\langle n|$ is an eigenvector of the superoperator $\mathcal{L}_{0 E}$ at eigenvalue $\lambda_{E}=0$. The corresponding eigenspace is infinitely degenerate. We denote by $P$ the projection operator over the $\lambda=0$ eigenspace, defined as

$$
P \rho=\rho_{\mathrm{St}} \otimes \sum_{n=0}^{\infty}|n\rangle\langle n| \operatorname{Tr}_{I}\{\langle n|\rho| n\rangle\},
$$

where $\operatorname{Tr}_{I}$ is the trace over the dipole and cavity degrees of freedom. At second order in $\eta$ one gets a closed equation for the center-of-mass dynamics of the form

$$
\begin{aligned}
\frac{d}{d t} \mu= & \eta^{2}\left[(S(\nu)+D)\left(b \mu b^{\dagger}-b^{\dagger} b \mu\right)\right. \\
& \left.+(S(-\nu)+D)\left(b^{\dagger} \mu b-b b^{\dagger} \mu\right)+\text { H.c. }\right],
\end{aligned}
$$

where $\mu=\operatorname{Tr}_{h}\{P \rho\}$ is the density matrix for the center-ofmass variables, obtained by tracing over the dipole and cavity degrees of freedom, and where the coefficients are given by

$$
D=\alpha \frac{\gamma}{2} \operatorname{Tr}_{I}\left\{\sigma^{\dagger} \sigma \rho_{\mathrm{St}}\right\}
$$

$$
\begin{aligned}
S(\nu) & =\frac{1}{\hbar^{2}} \int_{0}^{\infty} d \tau e^{\mathrm{i} \nu \tau} \operatorname{Tr}_{I}\left\{V_{1} e^{\mathcal{L}_{0 I} \tau} V_{1} \rho_{s S}\right\} \\
& =-\operatorname{Tr}_{I}\left\{V_{1}\left(\mathcal{L}_{0 I}+\mathrm{i} \nu\right)^{-1} V_{1} \rho_{S S}\right\} .
\end{aligned}
$$

In Eq. (26) we used

$$
\alpha=\int_{-1}^{1} \mathrm{~d} \cos \theta_{0} \cos ^{2} \theta_{0} \mathcal{N}\left(\cos \theta_{0}\right),
$$

which gives the angular dispersion of the atom momentum due to the spontaneous emission of photons. The operator $V_{1}$ in Eq. (27) is given by

$$
V_{1}=\varphi_{L} V_{L}+\varphi_{c} V_{c}
$$

where

$$
\begin{gathered}
V_{L}=\mathrm{i} \hbar \Omega\left(\sigma^{\dagger}-\sigma\right), \\
V_{c}=-\hbar \widetilde{g}\left(a \sigma^{\dagger}+a^{\dagger} \sigma\right)
\end{gathered}
$$

describe, respectively, the mechanical effects of the drive and of the cavity at first order in $\eta$, with the two coefficients

$$
\begin{gathered}
\varphi_{L}=\cos \theta_{L}, \\
\varphi_{c}=\cos \theta_{c} \tan \phi,
\end{gathered}
$$

which depend on the geometry of the setup. The operator (28) is the gradient of the atom-field interaction at the center of the trap and corresponds to the mechanical force in the semiclassical limit [40].

\section{Rate equation}

From Eq. (25) one can directly derive the rate equation for the occupation probability $p_{n}=\langle n|\mu| n\rangle$ of the phonon number state $|n\rangle$-namely,

$$
\frac{d}{d t} p_{n}=\eta^{2}\left\{(n+1) A_{-} p_{n+1}-\left[(n+1) A_{+}+n A_{-}\right] p_{n}+n A_{+} p_{n-1}\right\},
$$

where

$$
A_{ \pm}=2 \operatorname{Re}\{S(\mp \nu)+D\}
$$

are the rate of heating $\left(A_{+}\right)$and cooling $\left(A_{-}\right)$. The solution of this type of equation is well known [34]. The average phonon number obeys the equation

$$
\langle\dot{n}\rangle=-\eta^{2}\left(A_{-}-A_{+}\right)\langle n\rangle+\eta^{2} A_{+},
$$

which, for $A_{-}>A_{+}$, has solution

$$
\langle n\rangle_{t}=\langle n\rangle_{0} \mathrm{e}^{-W t}+\langle n\rangle_{\mathrm{St}}\left(1-\mathrm{e}^{-W t}\right) .
$$

Here, $\langle n\rangle_{0}$ is the initial average phonon number and

$$
\langle n\rangle_{\mathrm{St}}=\frac{A_{+}}{A_{-}-A_{+}}
$$

is the average phonon number at steady state, while

$$
W=\eta^{2}\left(A_{-}-A_{+}\right)
$$

is the cooling rate. 


\section{Discussion}

In Eq. (33) the internal dynamics enters through the coefficients $S(\nu)$ and $D$, which determine the rates (34). The function $S(\nu)$ is the spectrum of the fluctuations of the radiative force on the atom-namely, the Fourier transform of the autocorrelation function of the operator $V_{1}$ in Eq. (28). For the atom coupled to an optical resonator and driven transversally by a laser, we use the definition (28) in Eq. (27), and obtain

$$
S(\nu)=\varphi_{L}^{2} S_{L}(\nu)+\varphi_{c}^{2} S_{c}(\nu)+\varphi_{L} \varphi_{c} S_{c L}(\nu)
$$

Here,

$$
S_{L}=-\operatorname{Tr}_{\Lambda}\left\{V_{L}\left(\mathcal{L}_{0 I}+\mathrm{i} \nu\right)^{-1} V_{L} \rho_{\mathrm{St}}\right\}
$$

is the contribution of the mechanical effect due to the laser, the term

$$
S_{c}=-\operatorname{Tr}_{\Lambda}\left\{V_{c}\left(\mathcal{L}_{0 I}+\mathrm{i} \nu\right)^{-1} V_{c} \rho_{\mathrm{St}}\right\}
$$

the contribution of the mechanical effect due to the resonator, and

$$
S_{c L}=-\operatorname{Tr}_{I}\left\{V_{c}\left(\mathcal{L}_{0 I}+\mathrm{i} \nu\right)^{-1} V_{L} \rho_{\mathrm{St}}\right\}-\operatorname{Tr}_{I}\left\{V_{L}\left(\mathcal{L}_{0 I}+\mathrm{i} \nu\right)^{-1} V_{c} \rho_{\mathrm{St}}\right\}
$$

the contribution due to correlations between the mechanical effects of laser and resonator. Depending on the geometry of the setup, one term can be dominant over the others.

The coefficient $D$, Eq. (26), gives the diffusion in the dynamics of the center-of-mass motion. It is the product of two terms: the spontaneous emission rate of the excited state into the modes of the e.m. field and the stationary excitedstate population, which is determined by the overall dynamics at zeroth order in the Lamb-Dicke expansion.

\section{CAVITY COOLING OF TRAPPED ATOMS}

\section{A. Explicit form of the rate equation for cooling}

An analytical form for the rates entering Eq. (33) can be derived in the limit of a weak laser drive. The main steps of the derivation are reported in Appendix A. In this limit the heating and cooling rates take the form

$$
A_{ \pm}=\gamma \alpha\left|\mathcal{T}_{S}\right|^{2}+\gamma\left|\varphi_{L} \mathcal{T}_{L}^{\gamma, \pm}+\varphi_{c} \mathcal{T}_{c}^{\gamma, \pm}\right|^{2}+\kappa\left|\varphi_{L} \mathcal{T}_{L}^{\kappa, \pm}+\varphi_{c} \mathcal{T}_{c}^{\kappa, \pm}\right|^{2},
$$

with

$$
\begin{gathered}
\mathcal{T}_{S}=\Omega \frac{\delta_{c}+\mathrm{i} \kappa / 2}{f(0)}, \\
\mathcal{T}_{L}^{\gamma, \pm}=\mathrm{i} \Omega \frac{\left(\delta_{c} \mp \nu+\mathrm{i} \kappa / 2\right)}{f(\mp \nu)}, \\
\mathcal{T}_{L}^{\kappa, \pm}=\mathrm{i} \Omega \frac{\widetilde{g}}{f(\mp \nu)}, \\
\mathcal{T}_{c}^{\gamma, \pm}=-\Omega \frac{\widetilde{g}^{2}\left(2 \delta_{c} \mp \nu+\mathrm{i} \kappa\right)}{f(0) f(\mp \nu)},
\end{gathered}
$$

$$
\mathcal{T}_{c}^{\kappa, \pm}=-\Omega \frac{\widetilde{g}\left[(\Delta \mp \nu+\mathrm{i} \gamma / 2)\left(\delta_{c}+\mathrm{i} \kappa / 2\right)+\widetilde{g}^{2}\right]}{f(0) f(\mp \nu)},
$$

and

$$
f(x)=\left(x+\delta_{c}+\mathrm{i} \kappa / 2\right)(x+\Delta+\mathrm{i} \gamma / 2)-\widetilde{g}^{2} .
$$

The analytic form of Eqs. (39)-(44) allows for a more transparent reading of these complex dynamics, which can be mapped back to the processes shown in Fig. 4. The rates are the incoherent sum of three contributions: The first term $\gamma \alpha\left|\mathcal{T}_{S}\right|^{2}$ describes a change in the motional state by mechanical coupling to the modes external to the cavity-namely, by the recoil associated with the spontaneous emission of a photon. It corresponds to the process depicted in Fig. 4(a) and determines the diffusion coefficient through the relation

$$
D=\gamma \alpha\left|\mathcal{T}_{S}\right|^{2} / 2 \text {. }
$$

The second term $\gamma\left|\varphi_{L} \mathcal{T}_{L}^{\gamma, \pm}+\varphi_{c} \mathcal{T}_{c}^{\gamma, \pm}\right|^{2}$ describes scattering of a laser photon into the external modes by mechanical coupling to the laser $\left(\mathcal{T}_{L}\right)$ and to the cavity $\left(\mathcal{T}_{c}\right)$ field. The two transition amplitudes correspond to the processes depicted in Figs. 4(b) and 4(c), respectively. They add up coherently and may interfere. Note that these processes, together with the diffusive process, are dominant for $\kappa \ll \gamma$.

The third term $\kappa\left|\varphi_{L} \mathcal{T}_{L}^{\kappa, \pm}+\varphi_{c} \mathcal{T}_{c}^{\kappa, \pm}\right|^{2}$ describes scattering of a laser photon into the external modes of the electromagnetic field by cavity decay. The two amplitudes, appearing in this term, correspond to the processes depicted in Figs. 4(d) and 4(e), respectively. Also in this case they add up coherently and may interfere. This term is dominant for $\kappa \gg \gamma$.

Equation (39) contains the basic features of the dynamics of cavity cooling of trapped atoms. It has been derived (i) in the Lamb-Dicke regime, (ii) assuming that the electronic states are bound by the same center-of-mass potential, and (iii) in the limit in which the laser is a weak perturbation to atom and cavity dynamics. Moreover, it has been derived for one-dimensional motion. However, since at second order in $\eta$ the rate equations for the three directions of oscillation decouple in an anisotropic trap, it can be generalized to three-dimensional motion as it holds for any geometry of the setup. Below we show that this equation reproduces and generalizes results found in some particular regimes $[13,15]$. Moreover, Eq. (39) allows one to identify new parameter regimes characterized by novel dynamics that lead to efficient cooling. Some of these dynamics will be presented in Sec. V.

\section{B. Cooling in the bad-cavity limit}

Cooling in the bad-cavity limit, as discussed in [13], is recovered by maximizing the ratio $A_{-} / A_{+}$in the limit in which spontaneous emission is negligible. In Eq. (39) we set $\gamma=0$ and take $\left|\delta_{c}\right| \gg \nu$. The equivalence with the cooling and heating rates reported in [13] is evident by using the definitions $\widetilde{\delta}=\widetilde{g}^{2} \Delta_{c} /\left(\kappa^{2} / 4+\Delta_{c}^{2}\right)$ and $\tilde{\gamma}=\widetilde{g}^{2} \kappa /\left(\kappa^{2} / 4+\Delta_{c}^{2}\right)$, and imposing $\varphi_{c}=\varphi_{L}=1$ (namely, the cavity axes and the laser are parallel to the atomic motion). Below we use this notation but keep $\varphi_{L}$ and $\varphi_{c}$, thereby allowing for a more general geometry. From Eq. (39) we find 


$$
\begin{gathered}
A_{ \pm} \simeq \frac{\Omega^{2} \tilde{\gamma}}{\left[(\Delta-\tilde{\delta})^{2}+\tilde{\gamma}^{2} / 4\right]}\left|a_{ \pm}\right|^{2}, \\
a_{ \pm}=\varphi_{c}\left[1+\frac{2(\tilde{\delta}-\mathrm{i} \tilde{\gamma} / 2)}{\Delta-\tilde{\delta} \mp \nu+\mathrm{i} \tilde{\gamma} / 2}\right]-\mathrm{i} \varphi_{L} \frac{\Delta-\tilde{\delta}+\mathrm{i} \tilde{\gamma} / 2}{\Delta-\tilde{\delta} \mp \nu+\mathrm{i} \tilde{\gamma} / 2} .
\end{gathered}
$$

Here, two processes interfere-namely, the process in which the vibrational number changes by one phonon by absorbing a laser photon, depicted in Fig. 4(d) and described by the term $\mathcal{T}_{L}^{\kappa}$ in Eq. (39), and the process in which the vibrational number changes by one phonon by scattering a cavity photon, depicted in Fig. 4(e) and described by the term $\mathcal{T}_{c}^{\kappa}$ in Eq. (39). These dynamics are due to correlations between the mechanical effects of laser and the cavity, and they depend critically on the geometric setup, as is visible from Eq. (47).

\section{Sideband cooling in the good-cavity limit}

We consider the case where the atom is far-off resonance from the cavity and laser, $|\Delta| \gg \gamma, g, \kappa$. At leading order in $\Delta$ the rates of heating and cooling take the form

$$
A_{ \pm}=\frac{\Omega^{2}}{\Delta^{2}}\left[\left(\alpha+\varphi_{L}^{2}\right) \gamma+\frac{\widetilde{g}^{2} \kappa\left(\varphi_{L}^{2}+\varphi_{c}^{2}\right)}{\kappa^{2} / 4+\left(\delta_{c} \mp \nu\right)^{2}}\right]+O\left(1 / \Delta^{3}\right) .
$$

In this parameter regime there is no relevant contribution to the mechanical effects from correlations between the cavity and laser dipole force. Cooling is found for $\delta_{c}<0$, and the corresponding average phonon number at steady state is

$$
\langle n\rangle_{\mathrm{St}}^{(\Delta)}=\frac{\frac{\kappa^{2}}{4}+\left(\delta_{c}+\nu\right)^{2}}{4\left(-\delta_{c}\right) \nu}(1+B),
$$

with

$$
B=\frac{\gamma}{\widetilde{g}^{2} \kappa}\left(\frac{\alpha+\varphi_{L}^{2}}{\varphi_{L}^{2}+\varphi_{c}^{2}}\right)\left(\frac{\kappa^{2}}{4}+\left(\delta_{c}-\nu\right)^{2}\right)
$$

In the following we do not discuss the solutions leading to Doppler cooling and focus on the parameter regimes that lead to ground-state cooling, assuming $\gamma>\nu$.

For $\kappa \ll \nu$, Eq. (49) reaches the minimum value at $\delta_{c}=-\nu$,

$$
\left.\langle n\rangle_{\mathrm{St}}^{(\Delta)}\right|_{\delta_{c}=-\nu}=\frac{\kappa^{2}}{16 \nu^{2}}+\frac{1}{4 C_{1}}\left(\frac{\alpha+\varphi_{L}^{2}}{\varphi_{L}^{2}+\varphi_{c}^{2}}\right)\left(1+\frac{\kappa^{2}}{16 \nu^{2}}\right),
$$

where

$$
C_{1}=\widetilde{g}^{2} / \gamma \kappa
$$

is the one-atom cooperativity [41]. The corresponding cooling rate is

$$
\left.W\right|_{\delta_{c}=-\nu}=\eta^{2} 4 C_{1}\left(\varphi_{L}^{2}+\varphi_{c}^{2}\right) \frac{\Omega^{2}}{\Delta^{2}} \gamma\left(1-\frac{1}{1+(4 \nu / \kappa)^{2}}\right) .
$$

Therefore, large ground-state populations and large cooling rates can be achieved for $\nu \gg \kappa$ and $C_{1} \gg 1$-namely, for good cavities and in the limit in which the cavity linewidth is much smaller than the trap frequency.

Insight into these results can be found by using the dressed-state picture discussed in Sec. II: For $\kappa \ll \nu$, large $\Delta$, and $\delta_{c}=-\nu$ the excitation spectrum corresponds to the situation depicted in Fig. 3(a), where the the red sideband transition is resonant with the narrow resonance at frequency $\lambda_{+}$ $=\nu$, while the carrier and the blue sideband are driven far-off resonance. Hence, this condition is analogous to sideband cooling, whereby now the narrow resonance is the dressed state of the system composed by cavity and atom.

The cavity loss rate sets the lower limit to the width of the narrow resonance, on which sideband cooling is made, and thus to the efficiency of the process, as is visible in Eqs. (51) and (53). From these equations it is also visible that large cooperativities ensure better efficiencies.

The results reported in this section have been obtained from the rate equation (33) with the coefficients, Eqs. (39)-(44), expanded at leading order in $1 / \Delta$. Such an expansion is valid in the limit where the detuning between atom and cavity (respectively, laser) is the largest physical parameter. We remark that Eq. (51) is in agreement with the result reported in [15] in the corresponding parameter regime, whereby the different numerical factors, as well as the dependence on the angles, are due to the different laser configuration there considered.

\section{COOLING BY QUANTUM CORRELATIONS IN THE GOOD-CAVITY LIMIT}

In this section we present and discuss novel cooling dynamics based on quantum interference effects, which is dominant in the good-cavity limit, when

$$
\kappa \ll \nu, \gamma, \tilde{g} .
$$

In this regime we focus on the first two terms of the rates (39), corresponding to the processes in Figs. 4(a)-4(c) and treat cavity decay, giving rise to the processes depicted in Figs. 4(d) and 4(e), as small perturbations. In the following we assume that

$$
\phi \neq \frac{\pi}{2}(2 n+1)
$$

namely, the coupling to the cavity mode does not vanish at zeroth order in the Lamb-Dicke expansion and it is given by Eq. (23). Comparisons among the efficiencies of the cooling schemes are presented in Sec. VI.

\section{A. Discussion}

Efficient cooling is achieved by maximizing the rate $A_{-}$ together with the ratio $A_{-} / A_{+}$. In this way, ideally one maxi- 
mizes the cooling rate, Eq. (38), and minimizes the average number of phonons at steady state, Eq. (37). In general, by inspection of Eqs. (39)-(44) one can identify a strategy for maximizing $A_{-} / A_{+}$, consisting in identifying the parameters such that the heating and/or diffusion processes vanish. In this regime, the optimal parameters that maximize $A_{-}$are found whenever

$$
\operatorname{Re}\{f(\nu)\}=0,
$$

thereby minimizing the denominator of $A_{-}$. Physically, this corresponds to set the red sideband transition at a resonance of the atom-cavity system. This strategy is effective when the linewidth of the corresponding resonance is smaller than the trap frequency. Equation (54) leads to a condition that relates the cavity detuning $\delta_{c}$ with the atom detuning $\Delta$-namely,

$$
\Delta_{\mathrm{opt}}\left(\delta_{c}\right) \equiv \frac{\widetilde{g}^{2}+\gamma \kappa / 4}{\delta_{c}+\nu}-\nu,
$$

where we assume fixed couplings and decay rates, hence also a fixed cooperativity. For instance, in the case of sideband cooling discussed in Sec. IV C, the optimal cooling conditions are reached for $\delta_{c}=-\nu$, corresponding to the solution of Eq. (54) for $\Delta \rightarrow \infty$. In this limit, the linewidth of the dressedstate resonance which is used for cooling is infinitely small, and the steady-state occupation vanishes accordingly.

Below we discuss various regimes where ground-state cooling is efficient and which may be identified, for the corresponding parameter regimes, with an approximate solution of Eq. (54).

\section{B. Suppression of diffusion by quantum interference}

In this section we discuss a cooling scheme based on the suppression of diffusion by quantum interference. The cooling dynamics is based on the suppression of the carrier transition and can be understood with the dressed-state picture. As discussed in Sec. II, the carrier transition can vanish in the regime in which the laser and cavity are resonant. The sidebands due to the harmonic motion, however, are weak perturbations in opposition of phase with respect to the carrier. Thus, they give rise to photon scattering with probability given by the corresponding value of the excitation spectrum, depicted in Fig. 3(b) for some parameter regime. The cooling strategy is thus to enhance the red sideband over the blue sideband absorption, thereby profiting of the suppression of carrier excitations and thus of diffusion. This idea is reminiscent of cooling mechanisms based on quantum interference between atomic levels [30,37], whereby here the suppression of the carrier transition is due to the destructive interference between the laser and light elastically scattered into the resonator by the atom.

Diffusion is suppressed when $\mathcal{T}_{S}=0$ in Eq. (39), leading to the vanishing of the diffusion coefficient $D$, Eq. (26). From Eq. (39) this occurs when $\delta_{c}=0$ and, ideally, for $\kappa=0$. Let us first consider the ideal condition of a lossless resonator. In this case, for $\delta_{c}=0$ the steady-state average phonon number is given by

$$
\langle n\rangle_{\mathrm{St}}^{(0)}=\frac{\left[\nu(\nu+\Delta)-\widetilde{g}^{2}\right]^{2}+\gamma^{2} \nu^{2} / 4}{4 \nu \Delta\left(\widetilde{g}^{2}-\nu^{2}\right)} .
$$

Cooling is achieved when either the relations $\Delta>0$ and $\tilde{g}>\nu$ or the relations $\Delta<0$ and $\tilde{g}<\nu$ are fulfilled. The minimum for $\langle n\rangle_{\mathrm{St}}^{(0)}$ is obtained when $\Delta=\Delta_{\mathrm{opt}}\left(\delta_{c}=0\right)$; see Eq. (55). For these values the minimum number of phonon at steady state is

$$
\left.\langle n\rangle_{\mathrm{St}}^{(0)}\right|_{\Delta_{\mathrm{opt}}(0)}=\frac{\gamma^{2}}{16 \Delta_{\mathrm{opt}}(0)^{2}}=\frac{\gamma^{2} \nu^{2}}{16\left(\widetilde{g}^{2}-\nu^{2}\right)^{2}},
$$

with the corresponding cooling rate

$$
\left.W\right|_{\Delta_{\mathrm{opt}}(0)}=4 \eta^{2}\left(\varphi_{L}^{2}+\varphi_{c}^{2}\right) \frac{\Omega^{2}}{\gamma}\left(1-\frac{1}{1+\left[4 \Delta_{\mathrm{opt}}(0) / \gamma\right]^{2}}\right) .
$$

Therefore, ground-state cooling-namely, $\langle n\rangle_{\mathrm{St}}^{(0)} \ll 1$-is found when $\Delta=\Delta_{\text {opt }}$ and $|\Delta| \gg \gamma$ or, equivalently, for $\left|\widetilde{g}^{2} / \nu-\nu\right| \gg \gamma$. This condition can be fulfilled (i) when $\nu \gg \gamma$ and (ii) when $\widetilde{g}^{2} \gg \gamma \nu$, which is most interesting as it can give ground-state cooling even when $\nu \ll \gamma$. Below we discuss these two cases in detail.

Case (i) corresponds to the so-called strong confinement regime [28] — namely, when the linewidth of the dipole transition is smaller than the trap frequency. In this case sideband cooling is efficient in free space (i.e., in the absence of the resonator). Like for sideband cooling in free space, the implementation inside a cavity resonator requires $\Delta=-\nu$, leading to the final occupation $\langle n\rangle_{\mathrm{St}}^{(0)} \approx \gamma^{2} / 16 \nu^{2}$.

Case (ii) can be fulfilled in the so-called weak confinement regime [28] —namely, when the linewidth of the dipole transition is much larger than the trap frequency. The result (57) shows that ground-state cooling can be efficiently achieved. This is to our knowledge a novel regime. Here, $\Delta \sim \widetilde{g}^{2} / \nu$, so that we can rewrite the cooling limit as

$$
\left.\langle n\rangle_{\mathrm{St}}^{(0)}\right|_{\Delta_{\mathrm{opt}}(0)} \approx \gamma^{2} \nu^{2} / 16 \tilde{g}^{4} \text {. }
$$

We now discuss how the efficiency is modified for $\delta_{c}=0$ but $\kappa$ finite. At first order in $\kappa$ the diffusion coefficient $D=0$. In fact, $D=O\left(\kappa^{2}\right)$, being the stationary population of the excited state of second order in $\kappa$ in this regime $[32,33]$. At first order in this expansion the steady-state average phonon number is

$$
\langle n\rangle_{\mathrm{St}}=\langle n\rangle_{\mathrm{St}}^{(0)}(1+F),
$$

where the term

$$
F=\frac{\kappa^{2}}{\nu^{2}} C_{1} \frac{\gamma}{2} \mathcal{A}_{-}-2 \frac{\kappa}{\nu} \frac{\mathcal{A}_{-}}{\mathcal{A}_{-}-\mathcal{A}_{+}} \frac{\varphi_{L} \varphi_{c}}{\varphi_{L}^{2}+\varphi_{c}^{2}}
$$

is the correction at first order in $\kappa, C_{1}$ is the one-atom cooperativity defined in Eq. (52), and

$$
\mathcal{A}_{ \pm}=\frac{\nu^{2} \gamma}{\left[\nu(\nu \mp \Delta)-\widetilde{g}^{2}\right]^{2}+\nu^{2} \gamma^{2} / 4} .
$$

Cavity decay increases the linewidth of the dressed-state resonances and is thus detrimental. Nevertheless, for high 
cooperativities the result we find in Eq. (60) approaches the result of the ideal case, Eq. (59). In particular, for $\tilde{g} \gg \nu$ its value at $\Delta=\Delta_{\text {opt }}(0)$ takes the simple form

$$
\left.\left.\langle n\rangle_{\mathrm{St}}\right|_{\Delta_{\mathrm{opt}}(0)} \approx\langle n\rangle_{\mathrm{St}}^{(0)}\right|_{\Delta_{\mathrm{opt}}(0)}+\frac{1}{8 C_{1}},
$$

showing that the corrections scale with $1 / C_{1}$.

It must be remarked that the equations presented in this section have been obtained from Eqs. (39) in the limit of weak coupling. Nevertheless, they are also valid when the dipole is driven by a saturating laser field. In that case, at zeroth order in the Lamb-Dicke expansion the atom is in the ground state and the cavity in a coherent state with amplitude $\beta_{c}=-\Omega / \widetilde{g}$, such that the steady state at zeroth order is [31]

$$
\rho_{0 \mathrm{St}}=\left|g, \beta_{c}\right\rangle\left\langle g, \beta_{c}\right| .
$$

The derivation of the rate equation of cooling, obtained by making no assumption regarding the strength of the laser intensity, is reported in Appendix B. The result agrees with the results reported in this section, which have been evaluated from Eqs. (39) under the assumption of weak laser fields. This agreement is not a casuality: In fact, when the conditions for this interference effect are fulfilled, the atom is driven well below saturation even for strong laser and cavity fields, since they mutually cancel at the atomic position $[32,33]$. Nevertheless, the sideband transitions take place since they are out of phase with respect to the carrier.

\section{Suppression of heating by quantum interference}

In this section we discuss a cooling scheme based on the suppression of heating processes by quantum interference. This interference phenomenon is found in the good-cavity limit and corresponds to the situation in which the heating processes depicted in Figs. 4(b) and 4(c) cancel out. The corresponding parameters are identified in Eq. (39) by imposing the condition $\varphi_{L} \mathcal{T}_{L}^{\gamma,+}+\varphi_{c} \mathcal{T}_{c}^{\gamma,+}=0$. This condition can be fulfilled, for instance, when $\varphi_{L}=0$-namely, when the laser is orthogonal to the motional axis and therefore exerts no force- and $\mathcal{T}_{c}^{\gamma,+}=0$-namely, when the transitions to the blue sideband induced by the mechanical effects of the resonator vanish. Below we discuss this particular case.

We assume $\varphi_{L}=0$. Condition $\mathcal{T}_{c}^{\gamma,+}=0$ is fulfilled when $\delta_{c}=\nu / 2$ and, ideally, $\kappa=0$. In this limit $A_{+}=\gamma \alpha\left|\mathcal{T}_{S}\right|^{2}$ and the average phonon number at steady state is

$$
\langle n\rangle_{\mathrm{St}}^{(0)}=\alpha \frac{9 \gamma^{2} \nu^{2} / 16+\left[\widetilde{g}^{2}-3 \nu(\Delta+\nu) / 2\right]^{2}}{16 \widetilde{g}^{4} \varphi_{c}^{2}} .
$$

It reaches a minimum for $\Delta=\Delta_{\text {opt }}(\nu / 2)$-namely, $\Delta_{\text {opt }}(\nu / 2)$ $=2 \widetilde{g}^{2} / 3 \nu-\nu$-which has the form

$$
\left.\langle n\rangle_{\mathrm{St}}^{(0)}\right|_{\Delta_{\mathrm{opt}}(\nu / 2)}=\frac{9 \alpha}{16 \varphi_{c}^{2}} \frac{\gamma^{2} \nu^{2}}{16 \widetilde{g}^{4}}
$$

with the corresponding cooling rate

$$
\left.W\right|_{\Delta_{\mathrm{opt}}(\nu / 2)}=16 \eta^{2} \frac{\Omega^{2}}{\gamma} \frac{\varphi_{c}^{2}}{\left(1+3 \nu^{2} / 4 \widetilde{g}^{2}\right)^{2}+\left(3 \gamma \nu / 8 \widetilde{g}^{2}\right)^{2}} .
$$

To our knowledge, this is a novel cooling regime. Insight into this dynamics cannot be simply gained by inspection of the excitation rate at zeroth order in the Lamb-Dicke parameter. In fact, the disappearance of the blue sideband transition is due to a quantum interference effect between the paths of mechanical excitation driven by the resonator. Comparing this case with cooling by suppression of diffusion (see Sec. $\mathrm{V} \mathrm{B}$ ), one finds that for $\delta_{c}=\nu / 2$ one can reach lower temperatures in a faster time, as is evident from a comparison of Eqs. (66) and (67) with Eqs. (58) and (63) and with the results reported in Sec. IV C.

A finite, but small, value of $\kappa$ leads to the corrected average excitation

$$
\langle n\rangle_{\mathrm{St}}=\langle n\rangle_{\mathrm{St}}^{(0)}(1+\kappa F)+\kappa G,
$$

where

$$
\begin{gathered}
F=\frac{1}{2}\left[\frac{\widetilde{g}^{2} \gamma}{9 \gamma^{2} \nu^{2} / 16+\left[\widetilde{g}^{2}-3 \nu(\Delta+\nu) / 2\right]^{2}}\right. \\
\left.+\frac{\gamma^{2} / 4+(\Delta+\nu)^{2}}{\widetilde{g}^{2} \gamma}-\frac{2(\Delta+\nu)}{\gamma \nu}\right], \\
G=\frac{9 \gamma^{2} \nu^{2} / 16+\left[\widetilde{g}^{2}-3 \nu(\Delta+\nu) / 2\right]^{2}}{4 \widetilde{g}^{2} \gamma \nu^{2}}
\end{gathered}
$$

are the corrections at first order in $\kappa$. They lower the efficiency of the mechanism. In particular, the optimal final occupation number becomes

$$
\left.\langle n\rangle_{\mathrm{St}}\right|_{\Delta_{\mathrm{opt}}(\nu / 2)}=\left.\left(1+\frac{1}{8 C_{1}}\right)\langle n\rangle_{\mathrm{St}}^{(0)}\right|_{\Delta_{\mathrm{opt}}(\nu / 2)}+\frac{\alpha / \varphi_{c}^{2}+9}{64 C_{1}},
$$

while the corrections to the cooling rate scale with $1 / C_{1}$. Therefore, for large cooperativities this interference effect is relevant to the cooling dynamics. We remark that as for $\delta_{c}=\nu / 2$ the heating transition vanishes; similarly for $\delta_{c}=-\nu / 2$, the cooling transition cancels out.

\section{Suppression of diffusion and heating by quantum interference}

We finally discuss a cooling scheme based on the suppression of both diffusion and heating transitions by quantum interference. Let us first consider suppression of the carrier excitation, which leads to a vanishing diffusion coefficient. This can be achieved by using a standing-wave drive, such that the trap center is at one of its nodes. Therefore, at zeroth order in the Lamb-Dicke expansion the atom does not scatter any photon and the cavity is thus empty. Photon scattering originates from the dynamics due to the finite size of the atomic wave packet, and it is thus a process of second order in the Lamb-Dicke expansion. In order to investigate these dynamics, we evaluate the heating and cooling rates entering 
the rate equation (33) by considering a new Hamiltonian, which is given by the operator (5) with the new laser-dipole coupling:

$$
H_{L}=\hbar \Omega \cos \left(k x \cos \theta_{L}+\phi_{L}\right)\left(\sigma^{\dagger}+\sigma\right) .
$$

The condition for which the trap is at a node of the laser standing wave corresponds to choosing

$$
\phi_{L}=\pi / 2 .
$$

For this value, the interaction with the laser vanishes at zeroth order in the Lamb-Dicke expansion and the steady state is the empty cavity field and the atom in the ground statenamely, $\rho_{\mathrm{St}}^{\prime}=\left|g, 0_{c}\right\rangle\left\langle g, 0_{c}\right|$. Note that no assumption has been made on the value of the Rabi frequency $\Omega$. By expanding Eq. (72) at first order in the Lamb-Dicke parameter, we obtain in place of the operator (28) the new interaction term

$$
V_{L}=-\hbar \Omega \varphi_{L}\left(\sigma^{\dagger}+\sigma\right) .
$$

In the rest of this section we will consider $\varphi_{L}=1$.

Following the lines of the derivation as in Sec. III B with the new definitions, we obtain the equation for the external dynamics, Eq. (25), where now

$$
D=0
$$

and

$$
S(\nu)=-\operatorname{Tr}_{I}\left\{V_{L}\left(\mathcal{L}_{0 I}^{\prime}+i \nu\right)^{-1} V_{L} \rho_{S t}^{\prime}\right\} .
$$

Here, $\mathcal{L}_{0 I}^{\prime}$ has the same form as $\mathcal{L}_{0 I}$ in Eq. (19), with $H_{0 L}=0$. Clearly, the disappearance of the diffusion term is due to the fact that there is no field at zeroth order in the Lamb-Dicke expansion.

The term (73), giving the mechanical action on the atomic motion, originates solely from scattering of laser photons. In fact, the mechanical effects of the resonator field appear at higher order in the Lamb-Dicke expansion. The cooling and heating rates $A_{ \pm}^{\prime}=2 \operatorname{Re}\{S(\mp \nu)\}$ take the form

$$
A_{ \pm}^{\prime}=\gamma\left|\mathcal{T}_{1 L}^{\gamma \pm}\right|^{2}+\kappa\left|\mathcal{T}_{1 L}^{\kappa \pm}\right|^{2}
$$

where now

$$
\begin{gathered}
\mathcal{T}_{1 L}^{\gamma, \pm}=\Omega \frac{\left(\delta_{c} \mp \nu+\mathrm{i} \kappa / 2\right)}{f(\mp \nu)}, \\
\mathcal{T}_{1 L}^{\kappa, \pm}=\Omega \frac{\tilde{g}}{f(\mp \nu)},
\end{gathered}
$$

and

$$
f(x)=\left(x+\delta_{c}+\mathrm{i} \kappa / 2\right)(x+\Delta+\mathrm{i} \gamma / 2)-\widetilde{g}^{2} .
$$

Hence, the transition amplitudes do not relevantly differ from the ones in Eqs. (40)-(44). However, no low saturation limit is needed in the derivation of these results.

We study now the cooling dynamics in an exemplary limit—namely, in the case of a very good resonator. We first consider an ideal resonator-namely, $\kappa=0$. We obtain

$$
\left.A_{ \pm}^{\prime}\right|_{\kappa=0}=\frac{\Omega^{2}\left(\delta_{c} \mp \nu\right)^{2} \gamma}{\gamma^{2}(\delta \mp \nu)^{2} / 4+\left[(\delta \mp \nu)(\Delta \mp \nu)-\widetilde{g}^{2}\right]^{2}} .
$$

Thus, when $\delta_{c}=\nu$, then the heating transition vanishes. Since the diffusion is also zero, then $A_{+}^{\prime}=0$ and

$$
\langle n\rangle_{\mathrm{St}}^{(0)}=o\left(\eta^{2}\right) \text {. }
$$

The corresponding cooling rate reaches the maximum value for $\Delta=\Delta_{\text {opt }}(\nu)$ - namely, $\Delta_{\text {opt }}(\nu)=\left(\widetilde{g}^{2}-2 \nu^{2}\right) / 2 \nu$-and takes the form

$$
W=4 \eta^{2} \frac{\Omega^{2}}{\gamma} .
$$

This result is exact for $\kappa=0$.

Finite values of $\kappa$ introduce corrections to the heating rate, which takes the form

$$
A_{+}^{\prime} \simeq \kappa \Omega^{2} / \widetilde{g}^{2}=\frac{\Omega^{2}}{\gamma} \frac{1}{C_{1}} .
$$

Correspondingly, the average number of phonons at steady state is

$$
\langle n\rangle_{\mathrm{St}} \simeq \frac{\left[2 \nu(\Delta+\nu)-\widetilde{g}^{2}\right]^{2}+\gamma^{2} \nu^{2}}{4 \nu^{2} \gamma^{2}} \frac{1}{C_{1}} .
$$

The minimum value of the number of excitations at steady state is found at $\Delta=\Delta_{\text {opt }}(\nu)$ and is given by

$$
\left.\langle n\rangle_{\mathrm{St}}\right|_{\Delta_{\mathrm{opt}}(\nu)}=1 / 4 C_{1} .
$$

This cooling dynamics is novel and corresponds to the situation in which the excitation pathways of the combined multilevel-atom-cavity system interfere destructively, thereby suppressing the blue sideband excitation. They are reminiscent of cooling schemes for multilevel atoms discussed in [42], where suppression of the carrier and blue sideband transitions is achieved by quantum interference between atomic excitations. In the case studied here, however, the mechanism which leads to suppression of the carrier transition is different from the one that leads to the suppression of the blue sideband transition, and both are due to the composite effect of cavity and laser on the atom. Moreover, the parameter regime here considered is one of several possible that can be identified by imposing the disappearance of the blue sidebands transition.

\section{RESULTS}

In this section we compare the cooling efficiencies in the various regime, as evaluated from the analytical results, and check the range of validity of the analytical calculations with a quantum Monte Carlo wave function method, where the full quantum dynamics of master equation (4) is simulated. We focus on the good-cavity limit, in particular on the parameters $\kappa \ll \nu \ll \gamma$.

\section{A. Plot of the analytical results}

The plots in Fig. 5 show the phonon number at steady state and the cooling rate for different geometries of the 


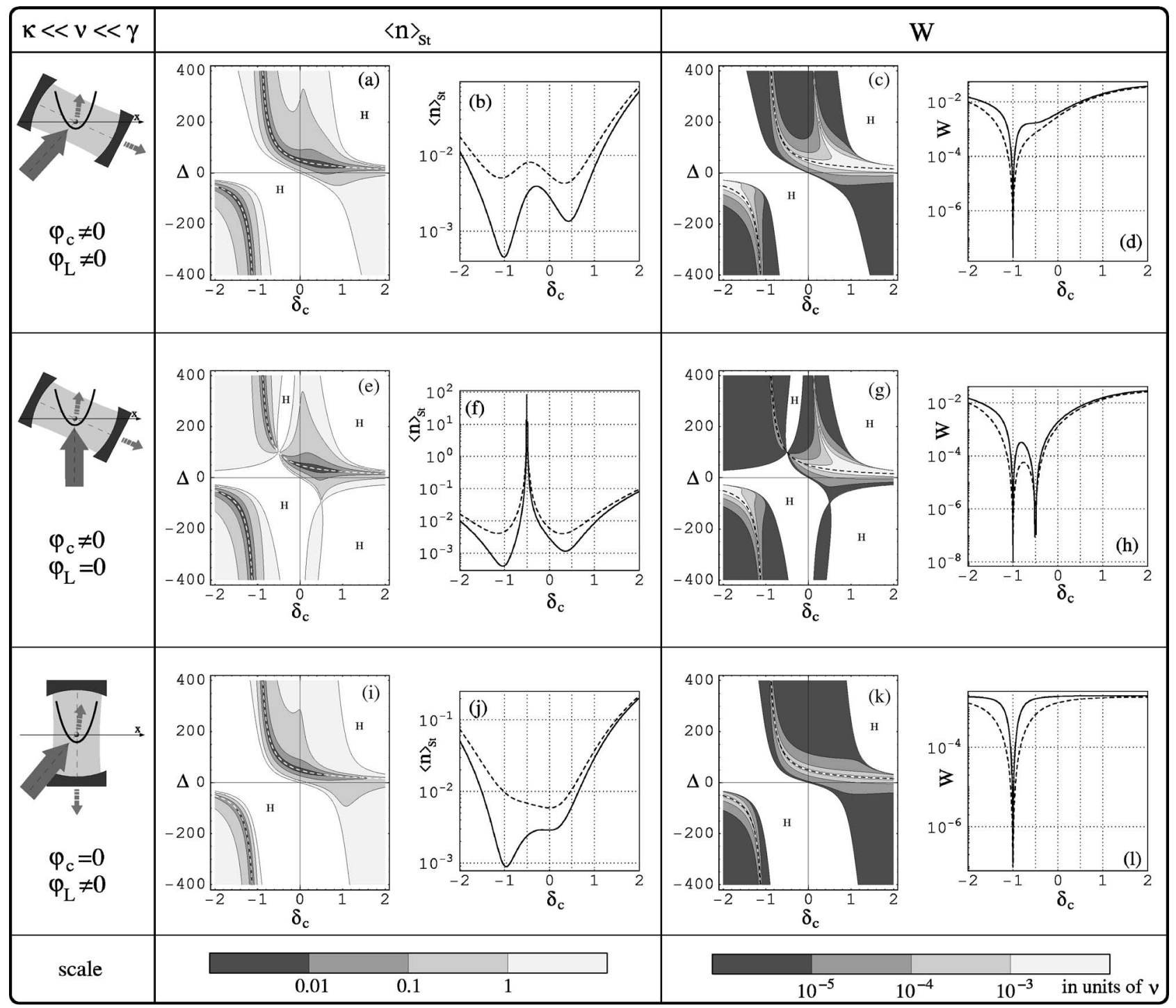

FIG. 5. Average phonon number at steady state $\langle n\rangle_{\mathrm{St}}$ and corresponding cooling rate $W$, in units of $\nu$, in the good-cavity limit, for $\kappa$ $\ll \nu \ll \gamma$ and for three possible geometries: in (a)-(d) (first row) both cavity and laser fields contribute to the cooling: here $\theta_{L}=\theta_{c}=\pi / 4$. In (e)-(h) (second row) the mechanical effects of the cavity solely determine cooling: here $\theta_{L}=\pi / 2$ and $\theta_{c}=\pi / 4$. In (i) $-(\mathrm{l})$ (last row) the mechanical effects of the laser solely determine cooling: here $\theta_{L}=\pi / 4$ and $\theta_{c}=\pi / 2$. The contour plots show $\langle n\rangle_{\mathrm{St}}$ and $W$ as a function of $\delta_{c}$ and $\Delta$ in units of $\nu$. The gradation of gray follows the scale where darkest region corresponds to the smallest values, the lightest region to the largest values. The corresponding values are reported at the bottom of the figure. The heating regions are not coded and explicitly indicated by the label $H$. The dashed curve appearing in each contour plot represents the curve $\Delta_{\text {opt }}\left(\delta_{c}\right)$, Eq. (55). The parameters are $\eta=0.1, \theta_{L}=\pi / 4, \Omega=\nu, \tilde{g}=7 \nu, \gamma=10 \nu$, and $\kappa=0.01 \nu$. The other plots display $\langle n\rangle_{\mathrm{St}}$ and $W$ as a function of $\delta_{c}$ and $\Delta_{\mathrm{opt}}\left(\delta_{c}\right)$, for the same parameters as of the contour plot and $\kappa=0.01 \nu$ (solid line) and $\kappa=0.1 \nu$ (dashed line).

setup. In particular, the plots of the first row depict the situation in which the mechanical effects on the atom are due to both laser and cavity field, the plots of the second row show the dynamics when the effects are solely due to the resonator, and the ones in the third row show the dynamics when the effects are solely due to the laser. The contour plots show most evidently the parameter regions where cooling is effective. Here, the dashed line represents the function (55) which determines the parameters minimizing the steady-state temperature. Clearly, in the neighborhood of this line the lowest temperature is achieved in all three cases. We note that the parameter regimes where cooling occurs may differ depend- ing on whether the dipole forces are due to the resonator or to the laser.

We now discuss the dynamics in detail. Due to the wealth of behaviors, we focus on the parameter regions where ground-state cooling appears efficient.

Figures 5(b), 5(f), and 5(j) display the value of the average phonon number as a function of $\delta_{c}$ and $\Delta_{\text {opt }}\left(\delta_{c}\right)$-namely, its value along the function (55). Figures 5(d), 5(h), and 5(1) show the corresponding cooling rates. Each plot displays two curves, which have been evaluated for two different values of the cavity decay rate $\kappa$ (solid curve, $\kappa=0.01 \nu$; dashed curve, $\kappa=0.1 \nu$ ). From these curves 
it is visible that, as the cavity decay rate increases, the cooling efficiency decreases; namely, the temperature gets higher and the cooling rate lower. Nevertheless, for the parameter here considered the cooling dynamics remains efficient. Let us now discuss the behavior as we vary $\delta_{c}$ and keep $\Delta=\Delta_{\text {opt }}\left(\delta_{c}\right)$.

In all cases the function $\langle n\rangle_{\mathrm{St}}$ exhibits a minimum at $\delta_{c}=-\nu$ at very large values of $\Delta$. This is the sideband cooling regime, discussed in Sec. IV C. The cooling rate at these points is very small, since it scales as $\Delta^{-2}$, as visible from Eq. (53) [43]. This cooling scheme exploits the dressed states of the system at zeroth order in the Lamb-Dicke expansion (see Sec. II), and its efficiency is thus relatively independent of whether the cavity or the laser forces determine cooling. Here, the cooling efficiency is very sensitive to variation of $\delta_{c}$, as visible from the contour plots. This sensitiveness is due to the narrowness of the linewidth of the dressed-state resonance which is used for cooling the motion.

The curves in Figs. 5(b) and 5(f) show also a minimum at $\delta_{c}=\nu / 2$. This is due to cooling by suppression of the resonator's mechanical coupling to the blue sideband transition (see Sec. V C). Clearly, this minimum is more enhanced in Fig. $5(\mathrm{f})$, where cooling is solely due to the mechanical effects of the cavity, and does not appear in Fig. 5(j), where the resonator field has no mechanical effects on the atom. The corresponding cooling rate is relatively large, the atom being driven close to resonance in this regime. An interesting characteristic, emerging from the contour plots, is that these cooling dynamics are relatively robust to fluctuations of the parameters, showing that ground-state cooling is efficient even in the regime in which suppression of the heating transition is partial.

The heating region at $\delta_{c}=-\nu / 2$, appearing in the case in which the mechanical effects of the cavity solely contribute to cooling, Figs. 5(e)-5(h), originates from the same interference effects that give rise to cooling at the value $\delta_{c}=\nu / 2$ and that for $\delta_{c}=-\nu / 2$ leads to suppression of the cooling transition (see Sec. V C).

Another regime where cooling is effective is found at $\delta_{c}=0$ when the mechanical effects are solely due to the laser. This parameter regime is characterized by small temperatures and large cooling rates, as visible from Figs. 5(i)-5(l). This is the regime in which the carrier transition is suppressed by an interference phenomenon at zeroth order in the motion (see Secs. II and V B). Cooling efficiency is robust against fluctuations of the parameters and appears to be relatively stable as the value of $\kappa$ is increased, as compared with the sideband cooling case [see Fig. 5(j)].

In general, we can conclude that in the range of values of $\delta_{c}$ around the interval $[0, \nu / 2]$, and close to atomic resonance, the cooling efficiency is relatively high. We remark that the final temperature is limited by the ratio $\kappa / \nu$. This is understood in the dressed-state picture, as the final limit to the narrow dressed-state resonance is set by the cavity decay rate $\kappa$.

\section{B. Numerical simulations}

The curves reported in Fig. 5 have been obtained from the analytical equations, which have been evaluated assuming
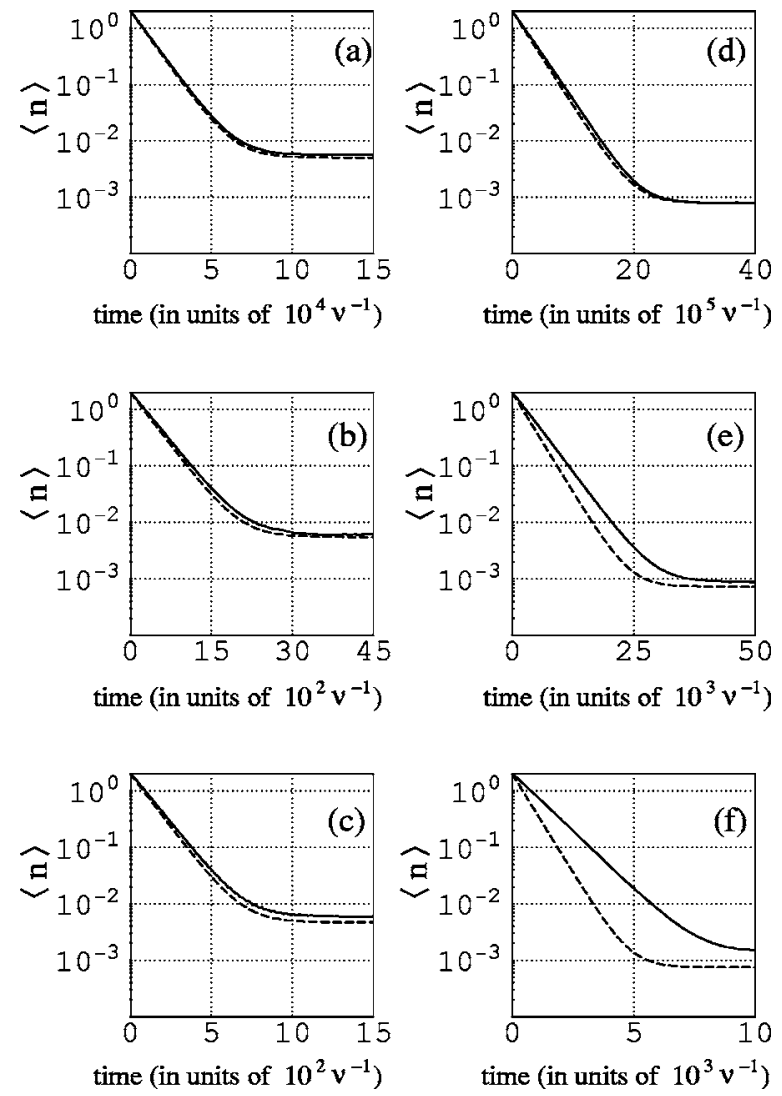

FIG. 6. Comparison between the analytical equations (33) and (39) and a full quantum Monte Carlo simulation of Eq. (4). The curves show the evolution of the average phonon number as the function of time in units of $\nu^{-1}$, the dashed lines correspond to the analytical predictions, and the solid lines correspond to the quantum Monte Carlo simulation. The parameters are $\eta=0.1, \theta_{L}=\theta_{c}=\phi$ $=\pi / 4, \Omega=\nu, \gamma=10 \nu$, and $\kappa=0.1 \nu$ and (a) $g=10 \nu, \delta_{c}=-1.1 \nu$; (b) $g=10 \nu, \delta_{c}=0$; (c) $g=10 \nu, \delta_{c}=0.5 \nu$; (d) $g=50 \nu, \delta_{c}=-1.1 \nu$; (e) $g=50 \nu, \delta_{c}=0$; and (f) $g=50 \nu, \delta_{c}=0.5 \nu$.

that dipole and cavity dynamics reach the steady state on a much faster time scale than the center-of-mass dynamics. In Fig. 6 we verify these results by comparing them with a full quantum Monte Carlo wave function simulation of master equation (4). We see that in general the analytical predictions are in agreement with the numerical results for a vast range of parameters, which are experimentally accessible. The discrepancies are small and are due to parameter regimes where the adiabatic evolution is not fulfilled. The discrepancies affect mostly the cooling rate, which varies by an overall factor, while the final average excitations of the center-of-mass oscillator are in agreement.

\section{CONCLUSIONS}

We have presented an extensive study of the cooling dynamics of trapped atoms in optical resonators. Our study is based on a rate equation, which we derive from the master equation of the system composed by atom and cavity, and whose validity is supported by numerical simulations taking into account the full quantum dynamics. Our analytical re- 
sults are valid in the Lamb-Dicke regime and when the center-of-mass potential is independent of the internal state, like in $[7,25,26]$.

The equations we derive reproduce the results reported in $[13,15]$ in their specific parameter regimes. Moreover, they allow us to identify new parameter regimes where the dynamics of the atomic center of mass, coupled to a laser and a cavity field, results from a nontrivial competition of the laser and of the resonator dipole forces, which can mutually interfere. These interference effects are at the basis of novel cooling schemes, which we identify in this paper and which allow us to reach very large ground-state occupations. The corresponding dynamics is reminescent of cooling schemes exploiting interference in multilevel atomic transitions $[30,42,44]$.

It must be remarked that the interference effects discussed in this work base themselves on the discreteness of the spectrum of the mechanical excitations, which is the same for the dipolar ground and excited states. The dynamics will be substantially modified when the external potential depends on the internal state, as the spectroscopic properties of the atom are changed and with it the scattering cross section. Preliminary considerations are in [37] for the case of trapped multilevel atoms, while the effects of state-dependent mechanical potentials on the cavity field have been discussed in [45].

In this paper we have focused on the case of dipole transitions, which in free space do not allow one to apply sideband cooling. Here, ground-state cooling can be obtained in good resonators, whereby the final cooling efficiency is limited by the cavity decay rate. The bad-cavity case is contained in the analytical equations we have derived. In this limit the effect of the laser seems to be predominant, and for a general configuration the optimal cooling corresponds to standard cavity sideband cooling [46]. This differs strikingly from the good-cavity limit, where correlations between atom and resonator can lead to very efficient cooling. We remark that this dynamics is largely modified when the drive is set on the resonator.

To conclude, this work considers the cooling dynamics to the potential ground state of a single atom inside a resonator. In the future we will investigate how the collective dynamics of several atoms, confined in a resonator, influences the cooling efficiency and how this can be used to prepare quantum states of the system in a controlled way.

\section{ACKNOWLEDGMENTS}

The authors wish to thank Helmut Ritsch and Axel Kuhn for several stimulating discussions. Support from the ISTnetwork QGATES and of the Spanish Ministerio de Educación y Ciencia (Ramon-y-Cajal Grant No. 129170) is acknowledged.

\section{APPENDIX A: LIMIT OF A WEAK DRIVING FIELD}

We consider the limit when the atom is weakly drivennamely, when the Rabi frequency $\Omega$ is much smaller than all the other physical parameters that characterize the internal dynamics. We study the dynamics of the motion in perturba- tion theory in second order in $\Omega$ and $\eta$, and neglect the terms of order $\eta^{4} \Omega^{2}, \eta^{2} \Omega^{4}$ and higher.

At zeroth order in $\Omega$ the laser field is zero. Hence, at steady state the cavity is empty and the atom is in the ground state. The state of dipole and cavity is described by the density matrix

$$
\rho_{\mathrm{St}}^{\circ}=\left|g, 0_{c}\right\rangle\left\langle g, 0_{c}\right|,
$$

which is the solution of the equation $\mathcal{L}_{0 I}^{\circ} \rho_{\mathrm{St}}^{\circ}=0$, with

$$
\mathcal{L}_{0 I}^{\circ} \rho=\left.\mathcal{L}_{0 I}\right|_{\Omega=0} \rho=\frac{1}{\mathrm{i} \hbar}\left[H_{\mathrm{at}}+H_{\text {cav }}+H_{0 \mathrm{at}-\mathrm{cav}}, \rho\right]+\mathcal{K} \rho+\mathcal{L}_{0 s} \rho
$$

and is obtained from $\mathcal{L}$, Eq. (4), at zeroth order in $\eta$ and $\Omega$. Following the general procedure described in Sec. III B, we define the projector $P \rho=\left|g, 0_{c}\right\rangle\left\langle g, 0_{c}\left|\otimes \Sigma_{n}\right| n\right\rangle\langle n| \operatorname{Tr}_{I}\{\langle n|\rho| n\rangle\}$ into the eigenspace of the superoperator,

$$
\mathcal{L}_{0}^{\circ}=\mathcal{L}_{0 I}^{\circ}+\mathcal{L}_{0 E},
$$

namely, the Liouvillian at zero order in $\eta$ and $\Omega$, at the eigenvalue zero. The effect of the perturbation in $\eta$ and $\Omega$ is described by the equation

$$
P \dot{\rho}=\mathcal{L}_{P} P \rho,
$$

where we have eliminated the coupling with other subspaces, thereby obtaining

$$
\begin{gathered}
\mathcal{L}_{\mathcal{P}}=\sum_{n, m=0}^{\infty} \eta^{n} \frac{\Omega^{m}}{\widetilde{g}^{m}} \mathcal{L}_{n, m} \\
=\eta^{2} \frac{\Omega^{2}}{\widetilde{g}^{2}} \mathcal{L}_{2,2}+O\left(\eta^{4} \frac{\Omega^{2}}{\widetilde{g}^{2}}\right)+O\left(\eta^{2} \frac{\Omega^{4}}{\widetilde{g}^{4}}\right) .
\end{gathered}
$$

At the lowest nonvanishing order only the term $\mathcal{L}_{2,2}$ is relevant and

$$
\begin{gathered}
\mathcal{L}_{\mathcal{P}} \simeq \eta^{2} \frac{\Omega^{2}}{\widetilde{g}^{2}} \mathcal{L}_{2,2} \\
=\varphi_{L}^{2} \mathcal{L}_{L}+\varphi_{c}^{2} \mathcal{L}_{c}+\varphi_{c} \varphi_{L} \mathcal{L}_{c L}+\mathcal{L}_{\text {diff }} .
\end{gathered}
$$

The subscripts $L, c$, and $c L$ label the terms describing processes in which the mechanical effect on the atoms are due, respectively, to the laser, the cavity, and the cooperative action of the laser and cavity. They have the form

$$
\begin{gathered}
\mathcal{L}_{L}=-P \mathcal{L}_{1 L} \mathcal{L}_{0}^{\circ-1} \mathcal{L}_{1 L}, \\
\mathcal{L}_{c}=-P \mathcal{L}_{1 c} \mathcal{L}_{0}^{\circ-1} \mathcal{L}_{1 c} \mathcal{L}_{0}^{\circ-1} \mathcal{L}_{0 L} \mathcal{L}_{0}^{\circ-1} \mathcal{L}_{0 L} \\
-P \mathcal{L}_{1 c} \mathcal{L}_{0}^{\circ-1} \mathcal{L}_{0 L} \mathcal{L}_{0}^{\circ-1} \mathcal{L}_{1 c} \mathcal{L}_{0}^{\circ-1} \mathcal{L}_{0 L}, \\
\mathcal{L}_{c L}=P \mathcal{L}_{1 L} \mathcal{L}_{0}^{\circ-1} \mathcal{L}_{1 c} \mathcal{L}_{0}^{\circ-1} \mathcal{L}_{0 L}+P \mathcal{L}_{1 c} \mathcal{L}_{0}^{\circ-1} \mathcal{L}_{0 L} \mathcal{L}_{0}^{\circ-1} \mathcal{L}_{1 L} \\
+P \mathcal{L}_{1 c} \mathcal{L}_{0}^{\circ-1} \mathcal{L}_{1 L} \mathcal{L}_{0}^{\circ-1} \mathcal{L}_{0 L},
\end{gathered}
$$

where the terms which trivially vanish have been omitted. Here, 


$$
\mathcal{L}_{0 L} \rho=-\frac{i}{\hbar}\left[H_{0 L}, \rho\right]
$$

describes the laser-atom interaction at zeroth order in $\eta$ where $H_{0 L}$ is defined in Eq. (21) and

$$
\mathcal{L}_{1 L} \rho=-\eta \frac{i}{\hbar}\left[\left(b^{\dagger}+b\right) V_{L}, \rho\right]
$$

and

$$
\mathcal{L}_{1 c} \rho=-\eta \frac{i}{\hbar}\left[\left(b^{\dagger}+b\right) V_{c}, \rho\right]
$$

describe, respectively, the laser-atom and cavity-atom interactions at first order in $\eta$ where $V_{L}$ and $V_{c}$ are defined in Eqs. (29) and (30). The diffusion due to carrier excitation is given by

$$
\mathcal{L}_{\text {diff }}=P \mathcal{L}_{2 s} \mathcal{L}_{0}^{\circ-1} \mathcal{L}_{0 L} \mathcal{L}_{0}^{\circ-1} \mathcal{L}_{0 L}
$$

where

$$
\mathcal{L}_{2 s} \rho=\frac{\gamma \alpha}{2} \sigma\left[2\left(b+b^{\dagger}\right) \rho\left(b+b^{\dagger}\right)-\left(b+b^{\dagger}\right)^{2} \rho-\rho\left(b+b^{\dagger}\right)^{2}\right] \sigma^{\dagger}
$$

is the Liouvillian at second order in $\eta$ for the atomic spontaneous emission.

Tracing over the internal degree of freedom we obtain an equation for the density matrix $\mu=\operatorname{Tr}_{h}\{P \rho\}$ for the center-ofmass variables,

$$
\begin{aligned}
\operatorname{Tr}_{I}\left\{\mathcal{L}_{\text {diff }} P \rho\right\} & =\eta^{2}\left[D\left(b \mu b^{\dagger}-b^{\dagger} b \mu+b^{\dagger} \mu b-b b^{\dagger} \mu\right)+\text { H.c. }\right] \\
\operatorname{Tr}_{I}\left\{\mathcal{L}_{j} P \rho\right\}= & \eta^{2}\left[S_{j}(\nu)\left(b \mu b^{\dagger}-b^{\dagger} b \mu\right)+S_{j}(-\nu)\left(b^{\dagger} \mu b-b b^{\dagger} \mu\right)\right. \\
& + \text { H.c. }],
\end{aligned}
$$

with $j=\{L, c, c L\}$. The coefficients $D$ and $S_{j}$ are defined as

$$
D=\frac{\gamma \alpha}{2} \operatorname{Tr}_{I}\left\{\sigma^{\dagger} \sigma \mathcal{L}_{0 I}^{\circ-1} \mathcal{L}_{0 \Omega} \mathcal{L}_{0 I}^{\circ-1} \mathcal{L}_{0 \Omega} \rho_{S S}^{\circ}\right\}
$$

and

$$
\begin{gathered}
S_{L}(\nu)=-\operatorname{Tr}_{\{}\left\{V_{L}\left(\mathcal{L}_{0 I}^{\circ}+i \nu\right)^{-1} V_{L} \rho_{S S}^{\circ}\right\}, \\
S_{c}(\nu)=-\operatorname{Tr}_{I}\left\{V _ { c } ( \mathcal { L } _ { 0 I } ^ { \circ } + i \nu ) ^ { - 1 } \left[V_{c} \mathcal{L}_{0 I}^{\circ-1} \mathcal{L}_{0 L}\right.\right. \\
\left.\left.+\mathcal{L}_{0 L}\left(\mathcal{L}_{0 I}^{\circ}+i \nu\right)^{-1} V_{c}\right] \mathcal{L}_{0 I}^{\circ-1} \mathcal{L}_{0 L} \rho_{s t}^{\circ}\right\}, \\
S_{c L}(\nu)=\operatorname{Tr}_{I}\left\{V_{L}\left(\mathcal{L}_{0 I}^{\circ}+i \nu\right)^{-1} V_{c} \mathcal{L}_{0 I}^{\circ-1} \mathcal{L}_{0 L} \rho_{s t}^{\circ}\right. \\
+V_{c}\left(\mathcal{L}_{0 I}^{\circ}+i \nu\right)^{-1} \mathcal{L}_{0 L}\left(\mathcal{L}_{0 I}^{\circ}+i \nu\right)^{-1} V_{L} \rho_{s t}^{\circ} \\
\left.+V_{c}\left(\mathcal{L}_{0 I}^{\circ}+i \nu\right)^{-1} V_{L} \mathcal{L}_{0 I}^{\circ-1} \mathcal{L}_{0 L} \rho_{s t}^{\circ}\right\} .
\end{gathered}
$$

Setting $S(\nu)=\varphi_{L}^{2} S_{L}(\nu)+\varphi_{c}^{2} S_{c}(\nu)+\varphi_{c} \varphi_{L} S_{c L}(\nu)$ we find an equation of the same form as Eq. (25). The real parts of these terms are

$$
\begin{gathered}
\operatorname{Re}\{D\}=\gamma \alpha\left|\mathcal{T}_{S}\right|^{2} / 2, \\
\operatorname{Re}\left\{S_{L}(\mp \nu)\right\}=\left(\gamma\left|\mathcal{T}_{L}^{\gamma, \pm}\right|^{2}+\kappa\left|\mathcal{T}_{L}^{\kappa, \pm}\right|^{2}\right) / 2,
\end{gathered}
$$

$$
\begin{gathered}
\operatorname{Re}\left\{S_{c}(\mp \nu)\right\}=\left(\gamma\left|\mathcal{T}_{c}^{\gamma, \pm}\right|^{2}+\kappa\left|\mathcal{T}_{c}^{\kappa, \pm}\right|^{2}\right) / 2, \\
\operatorname{Re}\left\{S_{c L}(\mp \nu)\right\}=\left(\gamma \mathcal{T}_{L}^{\gamma, \pm} \mathcal{T}_{c}^{\gamma, \pm *}+\kappa \mathcal{T}_{L}^{\kappa, \pm} \mathcal{T}_{c}^{\kappa, \pm *}\right) / 2+\text { c.c. }
\end{gathered}
$$

where the coefficients $\mathcal{T}_{j}$ are given explicitly in Eqs. (40)-(44). Finally, heating and cooling rates are given by $A_{ \pm}=2 \operatorname{Re}\{S(\mp \nu)+D\}$ and their explicit dependence on the physical parameters is reported in Eq. (39).

\section{APPENDIX B: LIMIT OF SMALL CAVITY LOSS RATE}

In this appendix we discuss the derivation of the rate equation, in the limit of small cavity loss and $\delta_{c}=0$, by making no assumption over the laser Rabi frequency $\Omega$, which may saturate the atomic transition. The results we obtain in this appendix are valid provided that $\tilde{g} \neq 0$.

In order to derive the rate equation for the atomic motion we closely follow the general approach described in Sec. III B, where here we expand at second order in $\eta$ and at first order in $\kappa$. The limit of applications of the perturbative expansion are found after identifying the smallest rate determining the internal dynamics for $\kappa=\delta_{c}=0$. This is the width of the narrow resonance $\gamma_{-}$, which for sufficiently large values of $|\Delta|$ takes the form

$$
\gamma_{-} \sim \frac{\gamma}{4}\left(1-\frac{|\Delta|}{\sqrt{\Delta^{2}+4 \widetilde{g}^{2}}}\right)
$$

Therefore, an expansion in $\kappa$ and $\eta$ is possible provided that $\kappa \ll \gamma_{-}$and $\eta \varphi_{c} \widetilde{g}, \eta \varphi_{L} \Omega \ll \gamma_{-}$. In this regime, the internal dynamics at zeroth order are described by the Liouvillian

$$
\mathcal{L}_{00 I}=\left.\mathcal{L}_{0 I}\right|_{\kappa=0, \delta_{c}=0}=\frac{1}{\mathrm{i} \hbar}\left[H_{\mathrm{at}}+H_{0 \mathrm{at}-\mathrm{cav}}+H_{0 L}, \rho\right]+\mathcal{L}_{0 s} \rho
$$

and the steady state, which is solution of $\mathcal{L}_{00 I} \rho_{0 \mathrm{St}}=0$, is given by Eq. (64). The superoperator at zeroth order in $\eta$ and $\kappa$ is given by

$$
\mathcal{L}_{00}=\mathcal{L}_{00 I}+\mathcal{L}_{0 E}
$$

and the corresponding projector over the eigenspace at eigenvalue zero is $P \rho=\left|g, \beta_{c}\right\rangle\left\langle g, \beta_{c}\left|\otimes \Sigma_{n}\right| n\right\rangle\langle n| \operatorname{Tr}_{I}\{\langle n|\rho| n\rangle\}$. The dynamics of this subspace at the lowest relevant order in $\eta$ and $\kappa / \gamma_{-}$is $P \dot{\rho}=\mathcal{L}_{P} P \rho$ where

$$
\mathcal{L}_{P}=\sum_{n=0, m=0}^{\infty} \eta^{n} \frac{\kappa^{m}}{\gamma_{-}^{m}} \mathcal{L}_{n, m}
$$

At lowest order it has the form

$$
\mathcal{L}_{P} \simeq \eta^{2} \mathcal{L}_{2,0}+\eta^{2} \frac{\kappa}{\gamma_{-}} \mathcal{L}_{2,1}
$$

where

$$
\eta^{2} \mathcal{L}_{2,0}=-P \mathcal{L}_{1} \mathcal{L}_{00}^{-1} \mathcal{L}_{1},
$$




$$
\eta^{2} \frac{\kappa}{\gamma_{-}} \mathcal{L}_{2,1}=P \mathcal{L}_{1} \mathcal{L}_{00}^{-1} \mathcal{L}_{1} \mathcal{L}_{00}^{-1} \mathcal{K}+P \mathcal{L}_{1} \mathcal{L}_{00}^{-1} \mathcal{K} \mathcal{L}_{00}^{-1} \mathcal{L}_{1}
$$

with

$$
\mathcal{L}_{1}=-\eta \frac{i}{\hbar}\left[\left(b^{\dagger}+b\right) V_{1}, \rho\right]
$$

Liouvillian at first order in $\eta$ for the coupling between atom and electromagnetic field, and $V_{1}$ given by Eqs. (28). Note that we have omitted to write the terms which trivially vanish. Tracing over the internal degree of freedom we obtain the equation

$$
\dot{\mu}=\operatorname{Tr}_{T}\left\{\mathcal{L}_{P} \rho_{0 S S} \otimes \mu\right\}
$$

for the center-of-mass variables density matrix $\mu=\operatorname{Tr}_{I}\{P \rho\}$, whereby

$$
\begin{aligned}
\operatorname{Tr}_{h}\left\{\mathcal{L}_{2, \ell} P \rho\right\}= & \eta^{2}\left[S_{\ell}(\nu)\left(b \mu b^{\dagger}-b^{\dagger} b \mu\right)\right. \\
& \left.+S_{\ell}(-\nu)\left(b^{\dagger} \mu b-b b^{\dagger} \mu\right)+\text { H.c. }\right] .
\end{aligned}
$$

Here the index $\ell=\{0,1\}$ indicates the order of the expansion in $\kappa$. From this equation we can identify the coefficients of Eq. (25), and thus

$$
D=0
$$

and

$$
S(\nu)=S_{0}(\nu)+S_{1}(\nu),
$$

where

$$
\begin{aligned}
S_{0}(\nu)= & -\operatorname{Tr}_{I}\left\{V_{1}\left(\mathcal{L}_{00 I}+\mathrm{i} \nu\right)^{-1} V_{1} \rho_{0 S S}\right\}, \\
S_{1}(\nu)= & \operatorname{Tr}_{I}\left\{V _ { 1 } ( \mathcal { L } _ { 0 0 I } + \mathrm { i } \nu ) ^ { - 1 } \left[V_{1} \mathcal{L}_{00 I}^{-1} \mathcal{K}\right.\right. \\
& \left.\left.+\mathcal{K}\left(\mathcal{L}_{00 I}+\mathrm{i} \nu\right)^{-1} V_{1}\right] \rho_{0 S S}\right\} .
\end{aligned}
$$

Note that $D=o\left(\kappa^{2}\right)$, as in this system the population of the atomic excited states grows quadratically with $\kappa[32,33]$.

Heating and cooling rates are found from the relation $A_{ \pm}=2 \operatorname{Re}\left\{S_{0}(\mp \nu)+S_{1}(\mp \nu)\right\}$ and take the form

$$
A_{ \pm}=\Omega^{2} \mathcal{A}_{ \pm}\left(\varphi_{L}^{2}+\varphi_{c}^{2}+\xi_{\kappa}^{ \pm}\right)
$$

whereby $\left|\xi_{\kappa}^{ \pm}\right| \ll 1$. In particular,

$$
\mathcal{A}_{ \pm}=\frac{\nu^{2} \gamma}{\left[\nu(\nu \mp \Delta)-\widetilde{g}^{2}\right]^{2}+\nu^{2} \gamma^{2} / 4},
$$

with

$$
\xi_{\kappa}^{ \pm}=\frac{\kappa^{2}}{\nu^{2}} C_{1}\left(1-\frac{\gamma}{2} \mathcal{A}_{ \pm}\right)\left(\varphi_{L}^{2}+\varphi_{c}^{2}\right)-\frac{\varphi_{c}^{2}}{2 C_{1}}+\frac{\kappa}{\nu}\left(\frac{\Delta \nu}{\widetilde{g}^{2}} \mp 1\right) \varphi_{L} \varphi_{c}
$$

The result (B11) coincides with the one obtained from expanding Eq. (39) to the first order in $\kappa$ and with $\delta_{c}=0$, from which the results in Eqs. (58) and (60) have been obtained. Nevertheless, in deriving the rates (B11) we have made no assumption on the strength of the laser intensity.
[1] C. Cohen-Tannoudji, in Fundamental Systems in Quantum Optics, Proceedings of the Les Houches Summer School of Theoretical Physics, Session LIII, Les Houches, 1990, edited by J. Dalibard, J.-M. Raymond, and J. Zinn-Justin (NorthHolland, Amsterdam, 1992), Vol. 53, pp. 1-164.

[2] V. Vuletic and S. Chu, Phys. Rev. Lett. 84, 3787 (2000).

[3] P. Domokos and H. Ritsch, J. Opt. Soc. Am. B 20, 1098 (2003).

[4] P. W. H. Pinkse, T. Fisher, P. Maunz, and G. Rempe, Nature (London) 404, 365 (2000).

[5] C. J. Hood, T. W. Lynn, A. C. Doherty, and H. J. Kimble, Science 287, 1447 (2000).

[6] P. Maunz, T. Puppe, I. Schuster, N. Syassen, P. W. H. Pinkse, and G. Rempe, Nature (London) 428, 50 (2004).

[7] J. McKeever, J. R. Buck, A. D. Boozer, A. Kuzmich, H. C. Nägerl, D. M. Stamper-Kurn, and H. J. Kimble, Phys. Rev. Lett. 90, 133602 (2003).

[8] H. W. Chan, A. T. Black, and V. Vuletic, Phys. Rev. Lett. 90, 063003 (2003); A. T. Black, H. W. Chan, and V. Vuletic, Phys. Rev. Lett. 91, 203001 (2003).

[9] D. Kruse, C. von Cube, C. Zimmermann, and Ph. W. Courteille, Phys. Rev. Lett. 91, 183601 (2003); C. von Cube, S. Slama, D. Kruse, C. Zimmermann, Ph. W. Courteille, G. R. M. Robb, N. Piovella, and R. Bonifacio, ibid. 93, 083601 (2004); S. Slama, C. von Cube, B. Deh, A. Ludewig, C. Zimmermann, and Ph. W. Courteille, ibid. 94, 193901 (2005).
[10] B. Nagorny, Th. Elsässer, and A. Hemmerich, Phys. Rev. Lett. 91, 153003 (2003).

[11] P. Bushev, A. Wilson, J. Eschner, C. Raab, F. Schmidt-Kaler, C. Becher, and R. Blatt, Phys. Rev. Lett. 92, 223602 (2004).

[12] S. Nussmann, K. Murr, M. Hijlkema, B. Weber, A. Kuhn, and G. Rempe, Nat. Phys. 1, 122 (2005).

[13] J. I. Cirac, M. Lewenstein, and P. Zoller, Phys. Rev. A 51, 1650 (1995).

[14] P. Horak, G. Hechenblaikner, K. M. Gheri, H. Stecher, and H. Ritsch, Phys. Rev. Lett. 79, 4974 (1997); G. Hechenblaikner, M. Gangl, P. Horak, and H. Ritsch, Phys. Rev. A 58, 3030 (1998).

[15] V. Vuletic, H. W. Chan, and A. T. Black, Phys. Rev. A 64, 033405 (2001).

[16] S. J. van Enk, J. McKeever, H. J. Kimble, and J. Ye, Phys. Rev. A 64, 013407 (2001).

[17] P. Domokos and H. Ritsch, Phys. Rev. Lett. 89, 253003 (2002).

[18] P. Domokos, Th. Salzburger, and H. Ritsch, Phys. Rev. A 66, 043406 (2002)

[19] P. Domokos, A. Vukics, and H. Ritsch, Phys. Rev. Lett. 92, 103601 (2004).

[20] A. Beige, P. L. Knight, and G. Vitiello, New J. Phys. 7, 96 (2005).

[21] Th. Salzburger and H. Ritsch, Phys. Rev. Lett. 93, 063002 (2004). 
[22] K. Murr, J. Phys. B 36, 2515 (2003).

[23] J. A. Sauer, K. M. Fortier, M. S. Chang, C. D. Hamley, and M. S. Chapman, Phys. Rev. A 69, 051804(R) (2004).

[24] G. R. Guthöhrlein, M. Keller, K. Hayasaka, W. Lange, and H. Walther, Nature (London) 414, 49 (2001).

[25] M. Keller, B. Lange, K. Hayasaka, W. Lange, and H. Walther, Nature (London) 431, 1075 (2004).

[26] A. B. Mundt, A. Kreuter, C. Becher, D. Leibfried, J. Eschner, F. Schmidt-Kaler, and R. Blatt, Phys. Rev. Lett. 89, 103001 (2002).

[27] S. Zippilli and G. Morigi, Phys. Rev. Lett. 95, 143001 (2005).

[28] J. Eschner, G. Morigi, F. Schmidt-Kaler, and R. Blatt, J. Opt. Soc. Am. B 20, 1003 (2003).

[29] J. Dalibard and C. Cohen-Tannoudji, J. Opt. Soc. Am. B 11, 1707 (1985).

[30] G. Morigi, J. Eschner, and C. H. Keitel, Phys. Rev. Lett. 85, 4458 (2000); C. F. Roos, D. Leibfried, A. Mundt, F. SchmidtKaler, J. Eschner, and R. Blatt, ibid. 85, 5547 (2000); F. Schmidt-Kaler, J. Eschner, G. Morigi, C. Roos, D. Leibfried, A. Mundt, and R. Blatt, Appl. Phys. B: Lasers Opt. 73, 807 (2001).

[31] P. M. Alsing, D. A. Cardimona, and H. J. Carmichael, Phys. Rev. A 45, 1793 (1992).

[32] S. Zippilli, G. Morigi, and H. Ritsch, Phys. Rev. Lett. 93, 123002 (2004).

[33] S. Zippilli, G. Morigi, and H. Ritsch, Eur. Phys. J. D 31, 507
(2004)

[34] S. Stenholm, Rev. Mod. Phys. 58, 699 (1986).

[35] J. Javanainen, M. Lindberg, and S. Stenholm, J. Opt. Soc. Am. B 1, 111 (1984).

[36] J. I. Cirac, R. Blatt, P. Zoller, and W. D. Phillips, Phys. Rev. A 46, 2668 (1992).

[37] G. Morigi, Phys. Rev. A 67, 033402 (2003).

[38] M. Bienert, W. Merkel, and G. Morigi, Phys. Rev. A 69, 013405 (2004)

[39] H. J. Briegel and B.-G. Englert, Phys. Rev. A 47, 3311 (1993). For a review, see B.-G. Englert and G. Morigi, in Coherent Evolution in Noisy Environments, Lecture Notes in Physics, Vol. 611, edited by A. Buchleitner and K. Hornberger (Springer-Verlag, Berlin, 2002), p. 55, and references therein.

[40] G. Nienhuis, P. van der Straten, and S. Q. Shang, Phys. Rev. A 44, 462 (1991).

[41] H. J. Kimble, in Cavity Quantum Electrodynamics, edited by P. R. Berman (Academic Press, New York, 1994), p. 203.

[42] J. Evers and C. H. Keitel, Europhys. Lett. 68, 370 (2004).

[43] Ideally, for $\delta_{c}=-\nu$ we should take $\Delta \rightarrow \infty$. Here, we have taken a large but finite value, consistent with the expansion in $1 / \Delta$ presented in Sec. IV C.

[44] I. Marzoli, J. I. Cirac, R. Blatt, and P. Zoller, Phys. Rev. A 49, 2771 (1994).

[45] J. Leach and P. R. Rice, Phys. Rev. Lett. 93, 103601 (2004).

[46] S. Zippilli and G. Morigi (unpublished). 